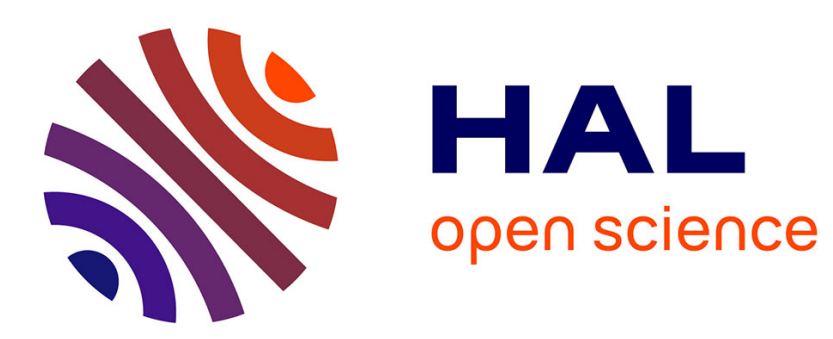

\title{
Besov regularity and new error estimates for finite volume approximations of the p-laplacian
}

Boris Andreianov, Franck Boyer, Florence Hubert

\section{To cite this version:}

Boris Andreianov, Franck Boyer, Florence Hubert. Besov regularity and new error estimates for finite volume approximations of the p-laplacian. Numerische Mathematik, 2005, 100 no 4, p. 565-592. 10.1007/s00211-005-0591-8 . hal-00004419

\section{HAL Id: hal-00004419 https://hal.science/hal-00004419}

Submitted on 11 Mar 2005

HAL is a multi-disciplinary open access archive for the deposit and dissemination of scientific research documents, whether they are published or not. The documents may come from teaching and research institutions in France or abroad, or from public or private research centers.
L'archive ouverte pluridisciplinaire HAL, est destinée au dépôt et à la diffusion de documents scientifiques de niveau recherche, publiés ou non, émanant des établissements d'enseignement et de recherche français ou étrangers, des laboratoires publics ou privés. 


\title{
Besov regularity and new error estimates for finite volume approximations of the p-laplacian
}

\author{
Boris Andreianov $^{1}$, Franck Boyer ${ }^{2}$, Florence Hubert ${ }^{2}$ \\ 1 Département de Mathématiques, Université de Franche-Comté, 16 route de \\ Gray, 25030 Besançon Cedex, France \\ 2 Laboratoire d'Analyse, Topologie et Probabilités, 39 rue F. Joliot Curie, 13453 \\ Marseille Cedex 13, France
}

The date of receipt and acceptance will be inserted by the editor

Summary In [1], we have constructed a family of finite volume schemes on rectangular meshes for the p-laplacian and we proved error estimates in case the exact solution lies in $W^{2, p}$. Actually, $W^{2, p}$ is not a natural space for solutions of the p-laplacian in the case $p>2$. Indeed, for general $L^{p^{\prime}}$ data it can be shown that the solution only belongs to the Besov space $B_{\infty}^{1+\frac{1}{p-1}, p}$.

In this paper, we prove Besov kind a priori estimates on the approximate solution for any data in $L^{p^{\prime}}$. We then obtain new error estimates for such solutions in the case of uniform meshes

Key words Finite volume methods - p-laplacian - Besov spaces

Mathematics Subject Classification (1991): 35J65 - 65N15 - 74S10

\section{Introduction}

In this paper we are interested in the finite volume numerical approximation on rectangular meshes of solutions to the p-laplacian with homogeneous Dirichlet boundary conditions $(1<p<+\infty)$ :

$$
\left\{\begin{array}{c}
-\operatorname{div}\left(|\nabla \bar{u}|^{p-2} \nabla \bar{u}\right)=f, \quad \text { on } \Omega, \\
\bar{u}=0, \text { on } \partial \Omega .
\end{array}\right.
$$

Correspondence to: fboyer@cmi.univ-mrs.fr 
In the sequel we assume that $f \in L^{p^{\prime}}(\Omega)$. The schemes we consider derive from the minimization of a discrete functional that approaches the functional associated to (1) defined by

$$
J: \bar{u} \mapsto \frac{1}{p} \int_{\Omega}|\nabla \bar{u}|^{p} d z-\int_{\Omega} f \bar{u} d z .
$$

These schemes are presented in Section 2 and the details of their construction are given in [1].

For these schemes, on rectangular non-uniform meshes, we proved in [1] the following error estimates

$$
\left\{\begin{aligned}
&\left\|\bar{u}^{\mathcal{T}}-u^{\mathcal{T}}\right\|_{1, p, \mathcal{T}} \leq C h\|\bar{u}\|_{W^{2, p}} \\
&+C h^{\frac{1}{p-1}}\|\bar{u}\|_{W^{2, p}}^{\frac{3 p-4}{p(p-1)}}\|f\|_{L^{p^{\prime}}}^{\frac{(p-2)^{2}}{p(p-1}}, \quad \text { for } p \geq 2 \\
&\left\|\bar{u}^{\mathcal{T}}-u^{\mathcal{T}}\right\|_{1, p, \mathcal{T}} \leq C h^{p-1}\|\bar{u}\|_{W^{2, p}}^{p-1}\|f\|_{L^{p^{\prime}}}^{\frac{2-p}{p-1}}, \text { for } p<2
\end{aligned}\right.
$$

as soon as the exact solution $\bar{u}$ lies in $W^{2, p}(\Omega)$. Here, $h$ is the size of the mesh $\mathcal{T}, u^{\mathcal{T}}$ is the approximate solution, $\bar{u}^{\mathcal{T}}$ the projection of the exact solution on the approximation space and $\|\cdot\|_{1, p, \mathcal{T}}$ stands for the discrete $W_{0}^{1, p}$ norm. All the precise definitions and notations are given in Section 2.1.

For $1<p \leq 2$, J.W. Barrett and W.B. Liu proved in [4] that if $f \in L^{p^{\prime}}(\Omega)$, then $\bar{u}$ lies in $H^{2}(\Omega)$ which is embedded in $W^{2, p}(\Omega)$. Hence, the error estimate (2) applies in this case. Unfortunately, for $p>2$, except in some particular situations, there seems to be no known condition on the regularity of the right-hand side $f$ ensuring that the solution $\bar{u}$ of (1) belongs to such a space.

In this paper, we address the problem of giving error estimates for less regular solutions of (1) that is to say for any data $f \in L^{p^{\prime}}(\Omega)$. Indeed, in this context, the regularity of $\bar{u}$ is given by the following result due to J. Simon in [11].

Theorem 1 If $\Omega$ is a rectangle and if $p>2$, then for any data $f$ in $L^{p^{\prime}}(\Omega)$, the solution $\bar{u}$ of $(1)$ belongs to the Besov space $B_{\infty}^{1+\frac{1}{p-1}, p}(\Omega)$ and, there exists a constant $C>0$ such that

$$
\|\bar{u}\|_{B_{\infty}^{1+\frac{1}{p-1}, p}} \leq C\|f\|_{L^{p^{\prime}}}^{\frac{1}{p-1}}, \quad \forall f \in L^{p^{\prime}}(\Omega) .
$$

We recall the definition of Besov spaces in Section 2.2.2 but for a thorough study of these spaces, we refer to [9,12]. Note that the assumption " $\Omega$ is a rectangle" is not necessary and the precise result given in [11] is valid in more general situations. Furthermore, Theorem 1 
is sharp: there exist solutions of (1) which belong to $B_{\infty}^{1+\frac{1}{p-1}, p}(\Omega)$, but do not belong to any space $W^{1+\frac{1}{p-1}+\varepsilon, p}(\Omega)$ or $B_{\infty}^{1+\frac{1}{p-1}+\varepsilon, p}(\Omega)$ with $\varepsilon>0$. We give an example of such solutions in Section 4 and we show the numerical results obtained for this solution whose regularity is critical for the problem.

In order to prove the error estimates, our approach here is radically different from the one we used in [1]. It is adapted from an idea of V.B. Tyukhtin [13] used by S. Chow in [6] in order to improve the error estimate obtained in [8] for the finite element approximation of solutions to the p-laplacian. Notice that in the finite element case, sharper error estimates for this problem are given by W.B. Liu and J.W. Barrett in [4] and [5].

The key-point of the method is to use the minimization properties of both continuous and discrete problems. To our knowledge, the use of this kind of techniques in the finite volume framework is new. This is possibly due to the fact that, unlike in the finite elements case, additional regularity assumptions on the exact solutions do not yield the corresponding "discrete" regularity properties for the finite volume approximate solutions (see Remark 4). Thus, the use of the natural regularity space (here $B_{\infty}^{1+\frac{1}{p-1}, p}(\Omega)$ ) is crucial for the adaptation of Chow's technique for finite volume methods.

Even though our results are expected to be true on general cartesian meshes, our proof in this paper only concerns the case of uniform meshes. More precisely, the crucial part of the proof (see Section 3.4) still holds for general meshes, but the proof of some a priori estimates (in particular in Lemma 4) can not be extended to this case in a straightforward way. In a forthcoming work, we will show that these a priori estimates can also be obtained for particular non-uniform cartesian grids allowing some refinement of the mesh near the singularities of the data.

The outline of this paper is the following. In Section 2, we present the finite volume schemes we are interested in and we introduce the notations used in the sequel. Section 3 is mainly devoted to the proof of the error estimate for solutions lying in the natural Besov space for the problem. We also give in Section 3.5 some improvements of our results in the case where the data $f$ lies in some Hölder space. Finally, we give some numerical results in Section 4 which show that our error estimate is sharp for solutions with the critical regularity for the problem. 


\section{The finite volume schemes}

\subsection{Notations}

Let $\Omega$ be a rectangular bounded domain of $\mathbb{R}^{2}$, without loss of generality we assume $\Omega=] 0, L_{x}[\times] 0, L_{y}[$. We consider a uniform mesh $\mathcal{T}$, i.e. a set of disjoint control volumes $\mathcal{K} \in \mathcal{T}$ isometric to a given reference rectangular volume $] 0, h[\times] 0, k\left[\right.$, such that $\bar{\Omega}=\cup_{\mathcal{K} \in \mathcal{T}} \overline{\mathcal{K}}$. We consider a family of meshes with $h$ tending to zero and that satisfy the following assumption:

$$
\exists c_{1}>0, \text { such that } c_{1} \leq \frac{k}{h} \leq \frac{1}{c_{1}} .
$$

We denote by $x_{\mathcal{K}}$ the center of the control volume $\mathcal{K}$. In order to take into account the boundary conditions, we introduce artificial points constructed by symmetry with respect to the boundaries of $\Omega$ (see Figure 1). The dual mesh $\mathcal{T}^{*}$ of $\mathcal{T}$ is defined to be the set of dual

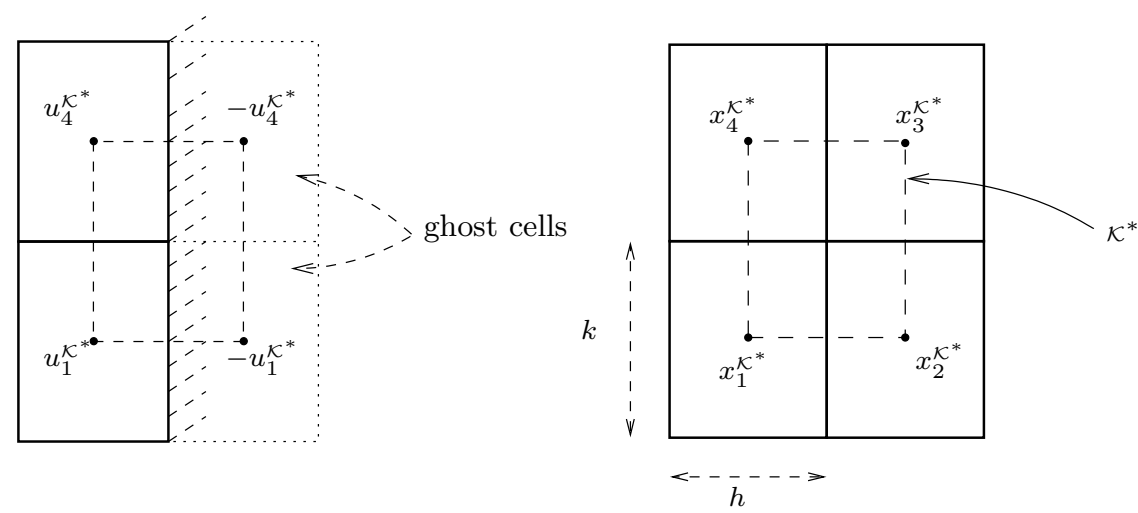

Fig. 1. Notations

rectangular control volumes whose vertices are the points $x_{\mathcal{K}}$ and the artifical points.

For any dual control volume $\mathcal{K}^{*}$,

- Let $\left(x_{i}^{\mathcal{K}^{*}}\right)_{i=1,2,3,4}$ be the vertices of the dual control volume $\mathcal{K}^{*}$ numbered counter clockwise starting from the lower left hand corner.

- Let $\left(\mathcal{K}_{i}^{\mathcal{K}^{*}}\right)_{i=1,2,3,4}$ be the corresponding control volumes with centers $\left(x_{i}^{\mathcal{K}^{*}}\right)_{i=1,2,3,4}$. 
- Finally let $l_{i}^{\mathcal{K}^{*}}$ be the distance between $x_{i}^{\mathcal{K}^{*}}$ and $x_{i+1}^{\mathcal{K}^{*}}$; in this paper, since the meshes are assumed to be uniform, we have $l_{1}^{\mathcal{K}^{*}}=l_{3}^{\mathcal{K}^{*}} \stackrel{\text { def }}{=} h$ and $l_{2}^{\mathcal{K}^{*}}=l_{4}^{\mathcal{K}^{*}} \stackrel{\text { def }}{=} k$ for any $\mathcal{K}^{*}$.

Conventionally, in a given dual control volume, the indices $i \in \mathbb{Z}$ are understood modulo 4.

The finite volume method associates to all control volumes $\mathcal{K}$ an unknown value $u_{\mathcal{K}}$. We denote the set $\left(u_{\mathcal{K}}\right)_{\mathcal{K} \in \mathcal{T}} \in \mathbb{R}^{\mathcal{T}}$ by $u^{\mathcal{T}}$. The discrete function $u^{\mathcal{T}}$ is called the approximate solution on the mesh $\mathcal{T}$. For any continuous function $v$ on $\Omega$, the discrete function $v^{\mathcal{T}}=$ $\left(v_{\mathcal{K}}\right)_{\mathcal{K} \in \mathcal{T}}$ with $v_{\mathcal{K}}=v\left(x_{\mathcal{K}}\right)$, is called the projection of $v$ on the space $\mathbb{R}^{\mathcal{T}}$ of discrete functions.

For a given discrete function $u^{\mathcal{T}} \in \mathbb{R}^{\mathcal{T}}$, the homogeneous Dirichlet boundary conditions are taken into account by using the ghost cells method (see Figure 1). That is, we extend the values of $u^{\mathcal{T}}$ on artificial points outside of $\Omega$ by odd symmetry with respect to the corresponding boundaries.

Given a dual control volume $\mathcal{K}^{*}$, we define the projection operator $T_{\mathcal{K}^{*}}$ which associates to each $u^{\mathcal{T}} \in \mathbb{R}^{\mathcal{T}}$ its values $T_{\mathcal{K}^{*}}\left(u^{\mathcal{T}}\right)=$ $\left(u_{1, \mathcal{K}^{*}}^{\mathcal{T}}, u_{2, \mathcal{K}^{*}}^{\mathcal{T}}, u_{3, \mathcal{K}^{*}}^{\mathcal{T}}, u_{4, \mathcal{K}^{*}}^{\mathcal{T}}\right)$ in the four control volumes $\left(\mathcal{K}_{i}^{\mathcal{K}^{*}}\right)_{i}$ that intersect $\mathcal{K}^{*}$. Note that for boundary dual control volumes, ghost cells are used in order to give sense to the definition of $T_{\mathcal{K}^{*}}$. For instance, if $\mathcal{K}^{*}$ is located at the right boundary of $\Omega$, we have by definition

$u_{2, \mathcal{K}^{*}}^{\mathcal{T}}=-u_{1, \mathcal{K}^{*}}^{\mathcal{T}}$ and $u_{3, \mathcal{K}^{*}}^{\mathcal{T}}=-u_{4, \mathcal{K}^{*}}^{\mathcal{T}}$, where $u_{1, \mathcal{K}^{*}}^{\mathcal{T}}=u_{\mathcal{K}_{1}^{\mathcal{K}}}, u_{4, \mathcal{K}^{*}}^{\mathcal{T}}=u_{\mathcal{K}_{4}^{\mathcal{K}}}$.

\subsection{Discrete norms}

2.2.1 Lebesgue and Sobolev norms Denote by $\mathbf{1}_{\mathcal{K}}$ the characteristic function of the control volume $\mathcal{K}$. Each discrete function $u^{\mathcal{T}} \in \mathbb{R}^{\mathcal{T}}$ is identified with the bounded function $u^{\mathcal{T}}=\sum_{\mathcal{K} \in \mathcal{T}} u_{\mathcal{K}} \mathbf{1}_{\mathcal{K}}$, so that for $r \in[1,+\infty]$ the norms $\left\|u^{\mathcal{T}}\right\|_{L^{r}}$ are naturally defined. Let us define a discrete Sobolev norm for the elements of $\mathbb{R}^{\mathcal{T}}$. For any $u^{\mathcal{T}} \in \mathbb{R}^{\mathcal{T}}$, and any $\mathcal{K}^{*} \in \mathcal{T}^{*}$, we define the differential quotients

$$
\delta_{i}^{\mathcal{K}^{*}}\left(u^{\mathcal{T}}\right)=\frac{u_{i+1, \mathcal{K}^{*}}^{\mathcal{T}}-u_{i, \mathcal{K}^{*}}^{\mathcal{T}}}{l_{i}^{\mathcal{K}^{*}}}, \quad i \in\{1, \ldots, 4\} .
$$

Definition 1 Consider $u^{\mathcal{T}} \in \mathbb{R}^{\mathcal{T}}$. For any $\mathcal{K}^{*}$, we define

$$
\left|u^{\mathcal{T}}\right|_{1, \mathcal{K}^{*}}=\left(\frac{1}{2} \sum_{i=1}^{4}\left|\delta_{i}^{\mathcal{K}^{*}}\left(u^{\mathcal{T}}\right)\right|^{2}\right)^{\frac{1}{2}},
$$


to be an approximation of $|\nabla \bar{u}|$, so that the discrete $W_{0}^{1, p}$ norm of $u^{\mathcal{T}}$ is defined by

$$
\left\|u^{\mathcal{T}}\right\|_{1, p, \mathcal{T}}=\left(\sum_{\mathcal{K}^{*} \in \mathcal{T}^{*}} m\left(\mathcal{K}^{*} \cap \Omega\right)\left|u^{\mathcal{T}}\right|_{1, \mathcal{K}^{*}}^{p}\right)^{\frac{1}{p}} .
$$

Lemma 1 (Discrete Poincaré inequality) Let $\mathcal{T}$ be a mesh of the rectangle $\Omega$. There exists a constant $C$ which only depends on $p$ such that for any $u^{\mathcal{T}} \in \mathbb{R}^{\mathcal{T}}$, we have

$$
\left\|u^{\mathcal{T}}\right\|_{L^{p}} \leq C \operatorname{diam}(\Omega)\left\|u^{\mathcal{T}}\right\|_{1, p, \mathcal{T}}
$$

The proof is an adaptation of the one given in [3].

2.2.2 Besov spaces and discrete Besov norms The whole family of Besov spaces is described in details for instance in $[9,12]$. For our purposes we only need to introduce, for any $0<\alpha<1$, the following space

$$
B_{\infty}^{1+\alpha, p}(\Omega)=\left(W^{1, p}(\Omega), W^{2, p}(\Omega)\right)_{\alpha, \infty},
$$

obtained from the classical Sobolev spaces using the $K$-method of real interpolation by Peetre (see [9]). The main point is that one can characterize the Besov space $B_{\infty}^{1+\alpha, p}(\Omega)$ using translation operators (see also [10]) as follows:

$$
\begin{aligned}
& B_{\infty}^{1+\alpha, p}(\Omega)=\left\{u \in W^{1, p}(\Omega),\right. \text { such that } \\
&\left.\sup _{r \in \mathbb{R}^{2}}\left(\int_{\Omega_{r}}\left|\frac{\nabla u(z+r)-\nabla u(z)}{|r|^{\alpha}}\right|^{p} d z\right)^{\frac{1}{p}}<+\infty\right\},
\end{aligned}
$$

where for any $r \in \mathbb{R}^{2}, \Omega_{r}=\{z \in \Omega, \quad[z, z+r] \subset \Omega\}$. An equivalent norm on this space is obtained by adding the $W^{1, p}$ norm and the supremum over $r \in \mathbb{R}^{2}$ above.

Let us introduce a discrete equivalent to this characterization in the case of general meshes.

Definition 2 (Discrete Besov norm) For any $\alpha \in] 0,1[$, we define the discrete $B_{\infty}^{1+\alpha, p}$ semi-norm for any $u^{\mathcal{T}} \in \mathbb{R}^{\mathcal{T}}$ by

$$
\left\|u^{\mathcal{T}}\right\|_{1+\alpha, p, \mathcal{T}}=\left(\sum_{\mathcal{K}^{*} \in \mathcal{T}^{*}} m\left(\mathcal{K}^{*} \cap \Omega\right)\left|\frac{\delta_{1}^{\mathcal{K}^{*}}\left(u^{\mathcal{T}}\right)+\delta_{3}^{\mathcal{K}^{*}}\left(u^{\mathcal{T}}\right)}{\left(l_{2, \mathcal{K}^{*}}\right)^{\alpha}}\right|^{p}\right)^{\frac{1}{p}},
$$

where $\delta_{i}^{\mathcal{K}^{*}}\left(u^{\mathcal{T}}\right)$ is defined in (4). The discrete $B_{\infty}^{1+\alpha, p}$ norm is then naturally defined by

$$
\left\|u^{\mathcal{T}}\right\|_{1+\alpha, p, \mathcal{T}}=\left\|u^{\mathcal{T}}\right\|_{1, p, \mathcal{T}}+\left\|u^{\mathcal{T}}\right\|_{1+\alpha, p, \mathcal{T}}
$$


Let us remark that we have $l_{1}^{\mathcal{K}^{*}} \delta_{1}^{\mathcal{K}^{*}}\left(u^{\mathcal{T}}\right)+l_{2}^{\mathcal{K}^{*}} \delta_{2}^{\mathcal{K}^{*}}\left(u^{\mathcal{T}}\right)+l_{3}^{\mathcal{K}^{*}} \delta_{3}^{\mathcal{K}^{*}}\left(u^{\mathcal{T}}\right)+$ $l_{4}^{\mathcal{K}^{*}} \delta_{4}^{\mathcal{K}^{*}}\left(u^{\mathcal{T}}\right)=0$ for any $u^{\mathcal{T}}$, so that, under the regularity assumptions of the meshes (3), we have

$$
\begin{aligned}
C_{1}\left\|u^{\mathcal{T}} \mid\right\|_{1+\alpha, p, \mathcal{T}} & \leq\left(\sum_{\mathcal{K}^{*} \in \mathcal{T}^{*}} m\left(\mathcal{K}^{*} \cap \Omega\right)\left|\frac{\delta_{2}^{\mathcal{K}^{*}}\left(u^{\mathcal{T}}\right)+\delta_{4}^{\mathcal{K}^{*}}\left(u^{\mathcal{T}}\right)}{\left(l_{1, \mathcal{K}^{*}}\right)^{\alpha}}\right|^{p}\right)^{\frac{1}{p}} \\
& \leq C_{2}\left\|u^{\mathcal{T}}\right\|_{1+\alpha, p, \mathcal{T}} .
\end{aligned}
$$

Remark 1 Note that $\delta_{1}^{\mathcal{K}^{*}}$ is an approximation of $\partial_{x} \bar{u}$ at the bottom of the dual control volume $\mathcal{K}^{*}$ and $\delta_{3}^{\mathcal{K}^{*}}$ is an approximation of $-\partial_{x} \bar{u}$ at the top of $\mathcal{K}^{*}$. As a consequence, the sum $\delta_{1}^{\mathcal{K}^{*}}+\delta_{3}^{\mathcal{K}^{*}}$ is, roughly speaking, an approximation of $\partial_{x} \bar{u}(\cdot)-\partial_{x} \bar{u}(\cdot+(0, h))$. Hence, the previous definition is a natural generalization of the Besov semi-norm introduced in (6).

In fact, the semi-norm we propose looks like an approximation of the $L^{p}$-norm of $\partial_{y}^{\alpha} \partial_{x} \bar{u}$, where $\partial_{y}^{\alpha}$ stands for the $\alpha$-th fractional derivative with respect to $y$. Thanks to (8), this semi-norm gives also the control of the $L^{p}$ norm of $\partial_{x}^{\alpha} \partial_{y} \bar{u}$. Nevertheless, this semi-norm is somewhat an incomplete approximation of the classical semi-norm in $B_{\infty}^{1+\alpha, p}(\Omega)$, since it lacks the control of terms like $\partial_{x}^{\alpha} \partial_{x} \bar{u}$ or $\partial_{y}^{\alpha} \partial_{y} \bar{u}$.

To cope with this problem, we may have considered the following discrete Besov norm

$$
\begin{aligned}
& \llbracket u^{\mathcal{T}} \rrbracket_{1+\alpha, p, \mathcal{T}}=\left\|u^{\mathcal{T}}\right\|_{1, p, \mathcal{T}} \\
& +\left(\sum_{\mathcal{K}^{*} \in \mathcal{T}^{*}} m\left(\mathcal{K}^{*} \cap \Omega\right) \sum_{\mathcal{L}^{*} \in V_{\mathcal{K}^{*}}} \sum_{i=1}^{4}\left|\frac{\delta_{i}^{\mathcal{K}^{*}}\left(u^{\mathcal{T}}\right)-\delta_{i}^{\mathcal{L}^{*}}\left(u^{\mathcal{T}}\right)}{\left(l_{i}^{\mathcal{K}^{*}}\right)^{\alpha}}\right|^{p}\right)^{\frac{1}{p}},
\end{aligned}
$$

where $V_{\mathcal{K}^{*}}$ is the set of neighbor dual control volumes of $\mathcal{K}^{*}$. Nevertheless, it appears that using the discrete Besov semi-norm (7) is sufficient to prove the error estimate we are interested in. That is the reason why all the intermediate results in the sequel are stated using (7) instead of (9).

Let us point out that we are able to derive a priori estimates for this more complete (and more complicated) norm (see Remarks 5 and 6), in particular the approximate solution satisfies a complete discrete Besov estimate.

\subsection{Description of the finite volume approximation}

The general form of symmetric, locally conservative finite volume schemes on cartesian meshes that are consistent with piecewise affine functions was described in [1]. 
In the particular case of uniform meshes, these schemes can be written as the following system of equations:

$$
\begin{aligned}
a\left(u^{\mathcal{T}}\right) \stackrel{\text { def }}{=}\left(a_{\mathcal{K}}\left(u^{\mathcal{T}}\right)\right)_{\mathcal{K} \in \mathcal{T}} \stackrel{\text { def }}{=} \sum_{\mathcal{K}^{*} \in \mathcal{T}^{*}} m\left(\mathcal{K}^{*} \cap \Omega\right) T_{\mathcal{K}^{*}}^{t} \circ a_{0} \circ T_{\mathcal{K}^{*}}\left(u^{\mathcal{T}}\right) \\
=\left(m(\mathcal{K}) f_{\mathcal{K}}\right)_{\mathcal{K} \in \mathcal{T}}
\end{aligned}
$$

where $u^{\mathcal{T}}=\left(u_{\mathcal{K}}\right)_{\mathcal{K} \in \mathcal{T}}$ are the unknowns and $f_{\mathcal{K}}$ denotes the mean value of the function $f$ on the control volume $\mathcal{K}$. Furthermore, the $\operatorname{map} a_{0}: \mathbb{R}^{4} \rightarrow \mathbb{R}^{4}$ is defined by:

$$
a_{0}(v) \stackrel{\text { def }}{=}(B v, v)^{\frac{p-2}{2}} B v, \quad \forall v \in \mathbb{R}^{4},
$$

where $B$ is a $4 \times 4$ symmetric matrix defined by the choice of one parameter $\xi$ as follows:

$$
B=\frac{1}{2 h k}\left(\begin{array}{cccc}
4 \xi+\frac{k}{h}+\frac{h}{k} & -4 \xi-\frac{k}{h} & 4 \xi & -4 \xi-\frac{h}{k} \\
-4 \xi-\frac{k}{h} & 4 \xi+\frac{k}{h}+\frac{h}{k} & -4 \xi-\frac{h}{k} & 4 \xi \\
4 \xi & -4 \xi-\frac{h}{k} & 4 \xi+\frac{k}{h}+\frac{h}{k} & -4 \xi-\frac{k}{h} \\
-4 \xi-\frac{h}{k} & 4 \xi & -4 \xi-\frac{k}{h} & 4 \xi+\frac{h}{h}+\frac{h}{k}
\end{array}\right),
$$

such that $(B v, v)^{1 / 2}$ is an approximation of the norm of the gradient of the solution. This choice ensures in particular the symmetry of the scheme.

Remark 2 In [1], we have studied more general schemes, where the parameter $\xi$ and consequently the matrix $B$ and the map $a_{0}$ may depend on $\mathcal{K}^{*}$. Even though our results in this paper are still true when the parameter $\xi=\xi_{\mathcal{K}^{*}}$ depends "continuously" on $\mathcal{K}^{*}$, we suppose for simplicity in the sequel that $\xi$ is constant.

Let us recall the following properties of these schemes (see [1]):

Definition 3 We say that a scheme defined by (10), (11), (12) is admissible if $B$ is a non-negative matrix that is to say if and only if we have:

$$
8 \xi+\frac{k}{h}+\frac{h}{k}>0
$$

Lemma 2 Let $\gamma>0$ be such that

$$
\xi \leq \frac{1}{\gamma} \text { and } 8 \xi+\frac{k}{h}+\frac{h}{k} \geq \gamma
$$

Then there exist $\beta_{1}, \beta_{2}>0$, depending only on $\gamma$ and on $c_{1}$ in (3) such that

$$
\beta_{1}\left|u^{\mathcal{T}}\right|_{1, \mathcal{K}^{*}} \leq\left|B^{\frac{1}{2}} T_{\mathcal{K}^{*}}\left(u^{\mathcal{T}}\right)\right| \leq \beta_{2}\left|u^{\mathcal{T}}\right|_{1, \mathcal{K}^{*}}, \forall \mathcal{K}^{*} \in \mathcal{T}^{*}, \forall u^{\mathcal{T}} \in \mathbb{R}^{\mathcal{T}} .
$$




\subsection{Discrete energy}

We call discrete energy of the scheme the following functional $J_{\mathcal{T}}$ acting on discrete functions $u^{\mathcal{T}} \in \mathbb{R}^{\mathcal{T}}$ :

$$
\begin{aligned}
J_{\mathcal{T}}\left(u^{\mathcal{T}}\right) & =\frac{1}{p}\left(a\left(u^{\mathcal{T}}\right), u^{\mathcal{T}}\right)-\sum_{\mathcal{K} \in \mathcal{T}} m(\mathcal{K}) f_{\mathcal{K}} u_{\mathcal{K}} \\
& =\frac{1}{p} \sum_{\mathcal{K}^{*} \in \mathcal{T}^{*}} m\left(\mathcal{K}^{*} \cap \Omega\right)\left|B^{\frac{1}{2}} T_{\mathcal{K}^{*}}\left(u^{\mathcal{T}}\right)\right|^{p}-\sum_{\mathcal{K} \in \mathcal{T}} m(\mathcal{K}) f_{\mathcal{K}} u_{\mathcal{K}} .
\end{aligned}
$$

It is proved in [1] that this functional is strictly convex and coercive and that its unique minimizing point is the unique solution of the discrete equations (10). Notice that this property is crucial since it gives a concrete way to compute the approximate solution of the problem.

Finally, the following properties hold:

Lemma 3 If $p \geq 2$, there exists a constant $C>0$ depending only on $c_{1}$ in (3) and $\gamma$ in (13), such that for any $u^{\mathcal{T}}, v^{\mathcal{T}} \in \mathbb{R}^{\mathcal{T}}$ we have

$$
J_{\mathcal{T}}\left(v^{\mathcal{T}}\right)-J_{\mathcal{T}}\left(u^{\mathcal{T}}\right)-\left(\nabla J_{\mathcal{T}}\left(u^{\mathcal{T}}\right), v^{\mathcal{T}}-u^{\mathcal{T}}\right) \geq C\left\|u^{\mathcal{T}}-v^{\mathcal{T}}\right\|_{1, p, \mathcal{T}}^{p},
$$

and

$$
\left(\nabla J_{\mathcal{T}}\left(v^{\mathcal{T}}\right)-\nabla J_{\mathcal{T}}\left(u^{\mathcal{T}}\right), v^{\mathcal{T}}-u^{\mathcal{T}}\right) \geq C\left\|u^{\mathcal{T}}-v^{\mathcal{T}}\right\|_{1, p, \mathcal{T}}^{p}
$$

Notice that this lemma is a discrete version of well-known properties for the continuous problem.

\section{Error estimate for Besov solutions}

When the solution $\bar{u}$ of (1) lies in the space $W^{2, p}(\Omega)$, we proved in [1] the error estimates (2). The main result of this paper is the following error estimate theorem which does not require any additional regularity assumption on $\bar{u}$.

Theorem 2 There exists a constant $C>0$ depending only on $c_{1}$ in (3) and $\gamma$ in (13), such that for any $f \in L^{p^{\prime}}(\Omega)$, the solution $\bar{u}$ of (1), and $u^{\mathcal{T}}$ solution of the scheme (10), satisfy

$$
\begin{gathered}
\left\|u^{\mathcal{T}}-\bar{u}^{\mathcal{T}}\right\|_{1, p, \mathcal{T}} \leq C h^{\frac{2}{p(p-1)}}\|f\|_{L^{p^{\prime}}}^{\frac{2}{p(p-1)}}\|\bar{u}\|_{W^{1, p}}^{1-\frac{2}{p}}, \quad \text { if } p \geq 3, \\
\left\|u^{\mathcal{T}}-\bar{u}^{\mathcal{T}}\right\|_{1, p, \mathcal{T}} \leq C h^{\frac{1}{p}}\|f\|_{L^{p^{\prime}}}^{\frac{1}{p}}\|\bar{u}\|_{W^{1, p}}^{\frac{1}{p}}, \quad \text { if } 2<p<3 .
\end{gathered}
$$


Remark 3 In the case where $2<p<3$, if the data $f$ is supposed to be in the Hölder space $\mathcal{C}^{0, \frac{3-p}{p-1}}(\bar{\Omega})$, then we can recover an error estimate in $h^{\frac{2}{p(p-1)}}$ (see Section 3.5).

We saw in [1] that the discrete Poincaré inequality holds true, so that we have

$$
\left\|u^{\mathcal{T}}-\bar{u}^{\mathcal{T}}\right\|_{L^{p}(\Omega)} \leq C\left\|u^{\mathcal{T}}-\bar{u}^{\mathcal{T}}\right\|_{1, p, \mathcal{T}}
$$

Furthermore, since $\bar{u} \in B_{\infty}^{1+\frac{1}{p-1}, p}(\Omega)$, we know (see [9]) that $\nabla \bar{u} \in$ $L^{\frac{2 p(p-1)}{p-2}}(\Omega)$ and then it is easy to prove that

$$
\left\|\bar{u}^{\mathcal{T}}-\bar{u}\right\|_{L^{p}(\Omega)} \leq C h\|\bar{u}\|_{B_{\infty}^{1+\frac{1}{p-1}, p}} \leq C h\|f\|_{L^{p^{\prime}}}^{\frac{1}{p-1}} .
$$

Hence, we have

$$
\left\|\bar{u}-u^{\mathcal{T}}\right\|_{L^{p}} \leq C\left\|u^{\mathcal{T}}-\bar{u}^{\mathcal{T}}\right\|_{1, p, \mathcal{T}}+C h\|f\|_{L^{p^{\prime}}}^{\frac{1}{p-1}},
$$

so that the theorem above also gives an intrinsic error estimate in $L^{p}(\Omega)$.

The sketch of the proof of this theorem is the following. In the first step, we derive a discrete Besov estimate for the approximate solution $u^{\mathcal{T}}$, which is a discrete version of Theorem 1 . In the second step, we prove a discrete Besov estimate for the projection $\bar{u}^{\mathcal{T}}$ of the exact solution $\bar{u}$ provided that $f \in L^{p^{\prime}}(\Omega)$. Thanks to these two estimates, we are able to prove the error estimates by using the minimization properties of $\bar{u}$ and $u^{\mathcal{T}}$ and the comparison Lemma 6 between the two functionals $J$ and $J_{\mathcal{T}}$.

Let us emphasize that in the finite elements framework, the discrete functional is nothing but the restriction of the continuous functional $J$ on a finite dimensional subspace of $W_{0}^{1, p}(\Omega)$. This is not the case in finite volume methods, thus it is needed to compare the continuous functional $J$ and the discrete functional $J_{\mathcal{T}}$ as it is made in Lemma 6.

Remark 4 In the finite volume case, the estimate $(2)$ for $W^{2, p}(\Omega)$ solutions cannot be improved using this kind of method, as S. Chow did in [6] in the finite elements framework. Indeed, our proof fundamentally relies on Lemma 6 applied to both the approximate solution $u^{\mathcal{T}}$ and to the projection of the exact solution $\bar{u}^{\mathcal{T}}$. Hence, the convergence order we can obtain depends essentially on the best discrete Besov estimate available for $u^{\mathcal{T}}$ and $\bar{u}^{\mathcal{T}}$. Unfortunately, it is clear that the best a priori estimate one can obtain for $u^{\mathcal{T}}$ is the one given by Lemma 4, even if the exact solution is supposed to be very smooth. 
In the sequel, let us denote by $C$ a generic constant that only depends on $p, c_{1}, \gamma$ and on $\Omega$.

\subsection{Discrete Besov estimate for the approximate solution}

Let us estimate the discrete Besov semi-norm for the solution $u^{\mathcal{T}}$ of the scheme (10). The proof is a discrete adaptation of the proof of Theorem 1 (see [11]).

Lemma 4 There exists a constant $C>0$ such that for any $f \in$ $L^{p^{\prime}}(\Omega)$, the approximate solution $u^{\mathcal{T}}$ of (1), defined by (10) satisfies

$$
\left\|u^{\mathcal{T}}\right\|_{1+\frac{1}{p-1}, p, \mathcal{T}} \leq C\|f\|_{L^{p^{\prime}}}^{\frac{1}{p-1}}
$$

Proof

- Step 1: Recall that we have $\Omega=] 0, L_{x}[\times] 0, L_{y}[$. Let us introduce $\widetilde{\Omega}=]-L_{x}, L_{x}[\times]-L_{y}, L_{y}[$, and $\tilde{\mathcal{T}}$ the unique uniform mesh on $\widetilde{\Omega}$ extending the mesh $\mathcal{T}$ on $\Omega$. It is clear that the dual mesh $\widetilde{\mathcal{T}}^{*}$ of $\widetilde{\mathcal{T}}$ is an extension of the dual mesh $\mathcal{T}^{*}$.

Let us denote by $u^{\widetilde{\mathcal{T}}}=\left(\tilde{u}_{\mathcal{K}}\right)_{\mathcal{K} \in \mathbb{R}^{\widetilde{T}}}$ the unique discrete function defined on the mesh $\widetilde{\mathcal{T}}$ which is odd with respect to both variables $x$ and $y$ and such that $u^{\widetilde{\mathcal{T}}}=u^{\mathcal{T}}$ on $\Omega$. It is natural to think of $u^{\widetilde{\mathcal{T}}}$ as a periodic discrete function with periodicity cell $\widetilde{\Omega}$.

It is clear that if we prove a local discrete Besov estimate for $u^{\widetilde{\mathcal{T}}}$ then we immediatly deduce the claim for $u^{\mathcal{T}}$.

By analogy with (10), we define:

$$
\left(m(\mathcal{K}) g_{\mathcal{K}}\right)_{\mathcal{K} \in \widetilde{\mathcal{T}}} \stackrel{\text { def }}{=} \sum_{\mathcal{K}^{*} \in \widetilde{\mathcal{T}}^{*}} m\left(\mathcal{K}^{*} \cap \widetilde{\Omega}\right) \widetilde{T}_{\mathcal{K}^{*}}^{t} \circ a_{0} \circ \widetilde{T}_{\mathcal{K}^{*}}\left(u^{\widetilde{\mathcal{T}}}\right),
$$

where $\widetilde{T}_{\mathcal{K}^{*}}$ is the projector from $\mathbb{R}^{\widetilde{\mathcal{T}}}$ onto $\mathbb{R}^{4}$ defined just like the projector $T_{\mathcal{K}^{*}}$ introduced in Section 2.1, but taking into account periodicity conditions (in both variables $x$ and $y$ ) instead of Dirichlet boundary conditions.

Note that because of the oddness with respect to $x$ and $y$ of $u^{\widetilde{\mathcal{T}}}$, the previous extension procedure is consistent with the ghost cells values we have used to take into account the boundary conditions in the scheme (see Figure 1).

It is now an easy computation, using (10), to prove that for any $\mathcal{K} \subset \Omega$, we have $g_{\mathcal{K}}=f_{\mathcal{K}}$.

- Step 2: Let us now prove that $\left(g_{\mathcal{K}}\right)_{\mathcal{K} \in \widetilde{\mathcal{T}}}$ is odd with respect to the variable $x$ for example (it is of course the same computation for 
the variable $y$ ). For any $\mathcal{K} \in \widetilde{\mathcal{T}}$ and $\mathcal{K}^{*} \in \widetilde{\mathcal{T}}^{*}$, we denote by $S \mathcal{K} \in \widetilde{\mathcal{T}}$ and $S \mathcal{K}^{*} \in \widetilde{\mathcal{T}}^{*}$ the volumes obtained by symmetry with respect to $\{x=0\}$. Furthermore, we recall that we have identified $\mathbf{1}_{\mathcal{K}}$ to the unique element of $\mathbb{R}^{\widetilde{\mathcal{T}}}$ which is zero on every control volume except on $\mathcal{K}$, where its value is 1 . Finally, let us introduce the matrix

$$
M=\left(\begin{array}{llll}
0 & 1 & 0 & 0 \\
1 & 0 & 0 & 0 \\
0 & 0 & 0 & 1 \\
0 & 0 & 1 & 0
\end{array}\right)
$$

Straightforward computations show that for $\mathcal{L}=S \mathcal{K}$ and $\mathcal{L}^{*}=S \mathcal{K}^{*}$, we have

$$
\widetilde{T}_{\mathcal{L}^{*}}\left(u^{\widetilde{\mathcal{T}}}\right)=-M \cdot \widetilde{T}_{\mathcal{K}^{*}}\left(u^{\widetilde{\mathcal{T}}}\right), \text { and } \widetilde{T}_{\mathcal{L}^{*}}\left(\mathbf{1}_{\mathcal{L}}\right)=M \cdot \widetilde{T}_{\mathcal{K}^{*}}\left(\mathbf{1}_{\mathcal{K}}\right) .
$$

Using (16) we deduce

$$
\begin{aligned}
m(\mathcal{L}) g_{\mathcal{L}} & =\sum_{\mathcal{L}^{*} \in \widetilde{\mathcal{T}}^{*}} m\left(\mathcal{L}^{*}\right)\left(\widetilde{T}_{\mathcal{L}^{*}}^{t} \circ a_{0} \circ \widetilde{T}_{\mathcal{L}^{*}}\left(u^{\widetilde{\mathcal{T}}}\right), \mathbf{1}_{\mathcal{L}}\right) \\
& =\sum_{\mathcal{L}^{*} \in \widetilde{\mathcal{T}}^{*}} m\left(\mathcal{L}^{*}\right)\left(a_{0}\left(\widetilde{T}_{\mathcal{L}^{*}}\left(u^{\widetilde{\mathcal{T}}}\right)\right), \widetilde{T}_{\mathcal{L}^{*}}\left(\mathbf{1}_{\mathcal{L}}\right)\right) \\
& =\sum_{\mathcal{K}^{*} \in \widetilde{\mathcal{T}}^{*}} m\left(\mathcal{K}^{*}\right)\left(a_{0}\left(-M \widetilde{T}_{\mathcal{K}^{*}}\left(u^{\widetilde{\mathcal{T}}}\right)\right), M \widetilde{T}_{\mathcal{K}^{*}}\left(\mathbf{1}_{\mathcal{K}}\right)\right) .
\end{aligned}
$$

From (12), we see that the matrices $B$ and $M$ commute so that we have, using $M^{t} M=\mathrm{Id}$ :

$$
\begin{aligned}
a_{0}\left(-M \widetilde{T}_{\mathcal{K}^{*}}\left(u^{\widetilde{\mathcal{T}}}\right)\right) & =-\left(B \widetilde{T}_{\mathcal{K}^{*}}\left(u^{\widetilde{\mathcal{T}}}\right), \widetilde{T}_{\mathcal{K}^{*}}\left(u^{\widetilde{\mathcal{T}}}\right)\right)^{\frac{p-2}{2}} M B \widetilde{T}_{\mathcal{K}^{*}}\left(u^{\widetilde{\mathcal{T}}}\right) \\
& =-M a_{0}\left(\widetilde{T}_{\mathcal{K}^{*}}\left(u^{\widetilde{\mathcal{T}}}\right)\right) .
\end{aligned}
$$

Therefore we have

$$
\begin{aligned}
m(\mathcal{L}) g_{\mathcal{L}} & =-\sum_{\mathcal{K}^{*} \in \widetilde{\mathcal{T}}^{*}} m\left(\mathcal{K}^{*}\right)\left(M a_{0}\left(\widetilde{T}_{\mathcal{K}^{*}}\left(u^{\widetilde{\mathcal{T}}}\right)\right), M \widetilde{T}_{\mathcal{K}^{*}}\left(\mathbf{1}_{\mathcal{K}}\right)\right) \\
& =-\sum_{\mathcal{K}^{*} \in \widetilde{\mathcal{T}}^{*}} m\left(\mathcal{K}^{*}\right)\left(a_{0}\left(\widetilde{T}_{\mathcal{K}^{*}}\left(u^{\widetilde{\mathcal{T}}}\right)\right), \widetilde{T}_{\mathcal{K}^{*}}\left(\mathbf{1}_{\mathcal{K}}\right)\right)=-m(\mathcal{K}) g_{\mathcal{K}} .
\end{aligned}
$$

As $m(\mathcal{K})=m(\mathcal{L})$, we deduce $g_{\mathcal{K}}=-g_{\mathcal{L}}$ provided that $\mathcal{L}=S \mathcal{K}$. This is exactly the claim.

In particular, we have proved that $\left(g_{\mathcal{K}}\right)_{\mathcal{K} \in \widetilde{\mathcal{T}}}$ is bounded in $L^{p^{\prime}}(\widetilde{\Omega})$ and more precisely we have

$$
\sum_{\mathcal{K} \in \widetilde{\mathcal{T}}} m(\mathcal{K})\left|g_{\mathcal{K}}\right|^{p^{\prime}}=4 \sum_{\mathcal{K} \in \mathcal{T}} m(\mathcal{K})\left|f_{\mathcal{K}}\right|^{p^{\prime}} \leq C\|f\|_{L^{p^{\prime}}(\Omega)} .
$$


- Step 3: Now it is enough to prove a discrete Besov estimate for the periodic problem (16).

For any $\mathcal{K} \in \widetilde{\mathcal{T}}$, we denote by $\tau_{h} \mathcal{K}$ (resp. $\left.\tau_{-h} \mathcal{K}\right)$ the right (resp. left) neighbor control volume of $\mathcal{K}$. Taking into account the periodicity conditions, $\tau_{h} \mathcal{K}$ and $\tau_{-h} \mathcal{K}$ are well defined for all $\mathcal{K}$. Let us define the translated discrete function $\tau_{h} u^{\widetilde{\mathcal{T}}}$ by

$$
\left(\tau_{h} u^{\widetilde{\mathcal{T}}}\right)_{\mathcal{K}}=\tilde{u}_{\tau_{h} \mathcal{K}}, \quad \forall \mathcal{K} \in \widetilde{\mathcal{T}}
$$

Using periodicity, we deduce from (16) that

$$
\left(m(\mathcal{K}) g_{\tau_{h} \mathcal{K}}\right)_{\mathcal{K}}=\sum_{\mathcal{K}^{*} \in \widetilde{\mathcal{T}}^{*}} m\left(\mathcal{K}^{*} \cap \widetilde{\Omega}\right) \widetilde{T}_{\mathcal{K}^{*}}^{t} \circ a_{0} \circ \widetilde{T}_{\mathcal{K}^{*}}\left(\tau_{h} u^{\widetilde{\mathcal{T}}}\right) .
$$

Subtracting (16), and taking the inner product in $\mathbb{R}^{\widetilde{\mathcal{T}}}$ with $\tau_{h} u^{\widetilde{\mathcal{T}}}-u^{\widetilde{\mathcal{T}}}$, we get

$$
\begin{aligned}
& \sum_{\mathcal{K}^{*} \in \widetilde{\mathcal{T}}^{*}} m\left(\mathcal{K}^{*} \cap \widetilde{\Omega}\right)\left(a_{0} \circ \widetilde{T}_{\mathcal{K}^{*}}\left(\tau_{h} u^{\widetilde{\mathcal{T}}}\right)-a_{0} \circ \widetilde{T}_{\mathcal{K}^{*}}\left(u^{\widetilde{\mathcal{T}}}\right), \widetilde{T}_{\mathcal{K}^{*}} \tau_{h} u^{\widetilde{\mathcal{T}}}-\widetilde{T}_{\mathcal{K}^{*}} u^{\widetilde{\mathcal{T}}}\right) \\
& =\sum_{\mathcal{K} \in \widetilde{\mathcal{T}}} m(\mathcal{K})\left(g_{\tau_{h} \mathcal{K}}-g_{\mathcal{K}}\right)\left(\widetilde{u}_{\tau_{h} \mathcal{K}}-\widetilde{u}_{\mathcal{K}}\right) \\
& =h \sum_{\mathcal{K} \in \widetilde{\mathcal{T}}} m(\mathcal{K}) g_{\mathcal{K}} \frac{\left(\tilde{u}_{\mathcal{K}}-\tilde{u}_{\tau_{-h} \mathcal{K}}\right)-\left(\tilde{u}_{\tau_{h} \mathcal{K}}-\tilde{u}_{\mathcal{K}}\right)}{h} \\
& \leq h\left(\sum_{\mathcal{K} \in \widetilde{\mathcal{T}}} m(\mathcal{K})\left|g_{\mathcal{K}}\right|^{p^{\prime}}\right)^{\frac{1}{p^{\prime}}}\left(\sum_{\mathcal{K} \in \widetilde{\mathcal{T}}} m(\mathcal{K})\left|\frac{\left(\tilde{u}_{\mathcal{K}}-\tilde{u}_{\tau_{-h} \mathcal{K}}\right)-\left(\tilde{u}_{\tau_{h} \mathcal{K}}-\tilde{u}_{\mathcal{K}}\right)}{h}\right|^{p}\right)^{\frac{1}{p}} \\
& \leq C h\|f\|_{L^{p^{\prime}}}\left(\sum_{\mathcal{K} \in \widetilde{\mathcal{T}}} m(\mathcal{K})\left|\frac{\left(\tilde{u}_{\mathcal{K}}-\tilde{u}_{\tau_{-h} \mathcal{K}}\right)-\left(\tilde{u}_{\tau_{h} \mathcal{K}}-\tilde{u}_{\mathcal{K}}\right)}{h}\right|^{p}\right)^{\frac{1}{p}} \cdot
\end{aligned}
$$

Using (14), the left-hand side member above is minorated by the quantity $C\left\|\tau_{h} u^{\widetilde{\mathcal{T}}}-u^{\widetilde{\mathcal{T}}}\right\|_{1, p, \widetilde{\mathcal{T}}}^{p}$. Hence, thanks to Definition 1 and to Lemma 3 the previous estimate gives

$$
\left\|\tau_{h} u^{\widetilde{\mathcal{T}}}-u^{\widetilde{\mathcal{T}}}\right\|_{1, p, \widetilde{\mathcal{T}}}^{p} \leq C h\|f\|_{L^{p^{\prime}}}\left\|\tau_{h} u^{\widetilde{\mathcal{T}}}-u^{\widetilde{\mathcal{T}}}\right\|_{1, p, \widetilde{\mathcal{T}}},
$$

and so we get

$$
\left\|\tau_{h} u^{\widetilde{\mathcal{T}}}-u^{\widetilde{\mathcal{T}}}\right\|_{1, p, \widetilde{\mathcal{T}}} \leq C h^{\frac{1}{p-1}}\|f\|_{L^{p^{\prime}}}^{\frac{1}{p-1}}
$$


We claim that this inequality gives the result. Indeed, let us define $\Delta_{h} u^{\widetilde{\mathcal{T}}}=\tau_{h} u^{\widetilde{\mathcal{T}}}-u^{\widetilde{\mathcal{T}}}$, and let us consider a given dual control volume $\mathcal{K}^{*} \in \widetilde{\mathcal{T}}^{*}$, we have

$$
\left|\delta_{4}^{\mathcal{K}^{*}}\left(\Delta_{h} u^{\widetilde{\mathcal{T}}}\right)\right|=\left|\frac{\left(\widetilde{T}_{\mathcal{K}^{*}}\left(\Delta_{h} u^{\widetilde{\mathcal{T}}}\right)\right)_{4}-\left(\widetilde{T}_{\mathcal{K}^{*}}\left(\Delta_{h} u^{\widetilde{\mathcal{T}}}\right)\right)_{1}}{k}\right| \leq \sqrt{2}\left|\Delta_{h} u^{\widetilde{\mathcal{T}}}\right|_{1, \mathcal{K}^{*}}
$$

But, by definition of the translation operator $\tau_{h}$, we have

$$
\begin{aligned}
& \left(\widetilde{T}_{\mathcal{K}^{*}}\left(\Delta_{h} u^{\widetilde{\mathcal{T}}}\right)\right)_{1}=\left(\widetilde{T}_{\mathcal{K}^{*}}\left(u^{\widetilde{\mathcal{T}}}\right)\right)_{2}-\left(\widetilde{T}_{\mathcal{K}^{*}}\left(u^{\widetilde{\mathcal{T}}}\right)\right)_{1}, \\
& \left(\widetilde{T}_{\mathcal{K}^{*}}\left(\Delta_{h} u^{\widetilde{\mathcal{T}}}\right)\right)_{4}=\left(\widetilde{T}_{\mathcal{K}^{*}}\left(u^{\widetilde{\mathcal{T}}}\right)\right)_{3}-\left(\widetilde{T}_{\mathcal{K}^{*}}\left(u^{\widetilde{\mathcal{T}}}\right)\right)_{4},
\end{aligned}
$$

and then

$$
\left|\frac{\left(\widetilde{T}_{\mathcal{K}^{*}}\left(\Delta_{h} u^{\widetilde{\mathcal{T}}}\right)\right)_{4}-\left(\widetilde{T}_{\mathcal{K}^{*}}\left(\Delta_{h} u^{\widetilde{\mathcal{T}}}\right)\right)_{1}}{k}\right|=\left|\delta_{2}^{\mathcal{K}^{*}}\left(u^{\widetilde{\mathcal{T}}}\right)+\delta_{4}^{\mathcal{K}^{*}}\left(u^{\widetilde{\mathcal{T}}}\right)\right| .
$$

Hence, we get the result from the estimate (17).

Remark 5 We also have

$$
\left|\delta_{1}^{\mathcal{K}^{*}}\left(u^{\widetilde{\mathcal{T}}}\right)-\delta_{1}^{\tau_{h} \mathcal{K}^{*}}\left(u^{\widetilde{\mathcal{T}}}\right)\right|=\left|\delta_{1}^{\mathcal{K}^{*}}\left(\Delta_{h} u^{\widetilde{\mathcal{T}}}\right)\right| \leq \sqrt{2}\left|\Delta_{h} u^{\widetilde{\mathcal{T}}}\right|_{1, \mathcal{K}^{*}},
$$

where $\tau_{h} \mathcal{K}^{*} \in \widetilde{\mathcal{T}}^{*}$ is the right neighbor of $\mathcal{K}^{*}$. Hence, we can estimate the complete Besov norm (see Remark 1) as follows:

$$
\llbracket u^{\mathcal{T}} \rrbracket_{1+\frac{1}{p-1}, p, \mathcal{T}} \leq C\|f\|_{L^{p^{\prime}}}^{\frac{1}{p-1}}
$$

\subsection{Discrete Besov estimate for the exact solution}

Let us now consider $\bar{u}$, the unique solution in $W_{0}^{1, p}(\Omega)$ of problem $(1)$ with data $f \in L^{p^{\prime}}(\Omega)$ and $p>2$. We know (see Theorem 1 ) that $\bar{u}$ is in the Besov space $B_{\infty}^{1+\frac{1}{p-1}, p}(\Omega)$. The goal of this section is to prove that the projection $\bar{u}^{\mathcal{T}}$ of $\bar{u}$ on the mesh $\mathcal{T}$ satisfies the same discrete Besov estimate as (15). The precise result is the following.

Lemma 5 Assume that $p>2$ and let $\alpha \in] 0,1[$. There exists a constant $C>0$ such that for any $v \in W_{0}^{1, p}(\Omega) \cap B_{\infty}^{1+\alpha, p}(\Omega)$, we have

$$
\left\|v^{\mathcal{T}} \mid\right\|_{1+\alpha, p, \mathcal{T}} \leq C\|v\|_{B_{\infty}^{1+\alpha, p}},
$$

where $v^{\mathcal{T}}=\left(v\left(x_{\mathcal{K}}\right)\right)_{\mathcal{K}^{*} \in \mathcal{T}}$ is the projection of $v$ on $\mathbb{R}^{\mathcal{T}}$. 
Proof We can extend $v$ to the unique function $\tilde{v}$ defined on the whole space $\mathbb{R}^{2}$, odd with respect to both variables $x$ and $y$ and periodic with periodicity cell $\widetilde{\Omega}=]-L_{x}, L_{x}[\times]-L_{y}, L_{y}[$. As $v$ vanishes on $\partial \Omega$, it is easily seen that $\tilde{v}$ lies in the space $B_{\infty, l o c}^{1+\alpha, p}\left(\mathbb{R}^{2}\right)$.

Note that this extension is consistent with the ghost cell method we used to treat the boundary conditions in the scheme. Furthermore a local discrete Besov estimate for the function $\tilde{v}$ and its projection is clearly equivalent to the claim. Hence, it is enough to prove the result without taking into account the boundaries.

Consider now a given dual control volume $\mathcal{K}^{*} \in \mathcal{T}^{*}$. Changing coordinates we can assume that $\left.\mathcal{K}^{*}=\right] 0, h[\times] 0, k\left[\right.$. Recall that $x_{1}, \ldots, x_{4}$ are the four vertices of $\mathcal{K}^{*}$, where we dropped the superscript $\mathcal{K}^{*}$ in order to simplify the notations in this proof. Let us denote by $x_{0}=\left(\frac{h}{2}, \frac{k}{2}\right)$ the center of $\mathcal{K}^{*}$.

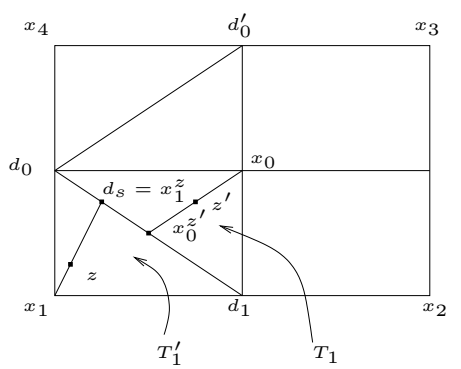

Fig. 2. Discrete Besov estimate in a dual control volume $\mathcal{K}^{*}$

For $s$ taking its values in $[0,1]$, the point $d_{s}=\left(\frac{h}{2} s, \frac{k}{2}(1-s)\right)$ describes the segment $\left[d_{0} d_{1}\right]$ as shown in Figure 2 . We denote by $T_{1}$ and $T_{1}^{\prime}$ the triangles $x_{0} d_{0} d_{1}$ and $x_{1} d_{0} d_{1}$, respectively.

Let us suppose that $v$ is smooth enough (the final result will follow by density). Using a Taylor expansion of $v$ between $x_{1}$ and $d_{s}$ and between $x_{0}$ and $d_{s}$, we get

$$
\begin{aligned}
& v\left(d_{s}\right)=v\left(x_{1}\right)+\int_{0}^{1} \nabla v\left(x_{1}+t\left(d_{s}-x_{1}\right)\right) \cdot\left(d_{s}-x_{1}\right) d t \\
& v\left(d_{s}\right)=v\left(x_{0}\right)+\int_{0}^{1} \nabla v\left(x_{0}+t\left(d_{s}-x_{0}\right)\right) \cdot\left(d_{s}-x_{0}\right) d t .
\end{aligned}
$$


Subtracting these two equalities and integrating with respect to $s$, we obtain

$$
\begin{aligned}
v\left(x_{1}\right)-v\left(x_{0}\right)= & -\int_{0}^{1} \int_{0}^{1} \nabla v\left(x_{1}+t\left(d_{s}-x_{1}\right)\right) \cdot\left(d_{s}-x_{1}\right) d t d s \\
& +\int_{0}^{1} \int_{0}^{1} \nabla v\left(x_{0}+t\left(d_{s}-x_{0}\right)\right) \cdot\left(d_{s}-x_{0}\right) d t d s
\end{aligned}
$$

Now, let us consider the change of variables $(s, t)$ into $z=x_{1}+t\left(d_{s}-\right.$ $\left.x_{1}\right)$ in the first term. Its Jacobian determinant is $-t \frac{h k}{4}$, and we have $t=\frac{\left|z-x_{1}\right|}{\left|d_{s}-x_{1}\right|}$ so that the first term becomes

$$
-\frac{4}{h k} \int_{T_{1}^{\prime}} \frac{\left|d_{s}-x_{1}\right|}{\left|z-x_{1}\right|} \nabla v(z) \cdot\left(d_{s}-x_{1}\right) d z .
$$

In this formula, $d_{s}$ is the intersection point of the segment $\left[d_{0} d_{1}\right]$ with the line passing through $z \in T_{1}^{\prime}$ and $x_{1}$. In the sequel, we will denote this point by $x_{1}^{z}$ (see Figure 2). With similar computations for the second term, we get

$$
\begin{aligned}
v\left(x_{1}\right)-v\left(x_{0}\right)= & -\frac{4}{h k} \int_{T_{1}^{\prime}} \frac{\left|x_{1}^{z}-x_{1}\right|}{\left|z-x_{1}\right|} \nabla v(z) \cdot\left(x_{1}^{z}-x_{1}\right) d z \\
& +\frac{4}{h k} \int_{T_{1}} \frac{\left|x_{0}^{z}-x_{0}\right|}{\left|z-x_{0}\right|} \nabla v(z) \cdot\left(x_{0}^{z}-x_{0}\right) d z,
\end{aligned}
$$

where $x_{z}^{0}$ is the intersection point between the line passing through $z \in T_{1}$ and $x_{0}$, and the segment $\left[d_{0} d_{1}\right]$.

Now let us apply the formula (18) to the translated function $\tau_{h, k} v$ : $z \mapsto v\left(z+\left(x_{0}-x_{1}\right)\right)$. We have $\tau_{h, k} v\left(x_{1}\right)=v\left(x_{0}\right)$ and $\tau_{h, k} v\left(x_{0}\right)=v\left(x_{3}\right)$ so that, it follows

$$
\begin{aligned}
v\left(x_{0}\right)-v\left(x_{3}\right)= & -\frac{4}{h k} \int_{T_{1}^{\prime}} \frac{\left|x_{1}^{z}-x_{1}\right|}{\left|z-x_{1}\right|} \nabla\left(\tau_{h, k} v\right)(z) \cdot\left(x_{1}^{z}-x_{1}\right) d z \\
& +\frac{4}{h k} \int_{T_{1}} \frac{\left|x_{0}^{z}-x_{0}\right|}{\left|z-x_{0}\right|} \nabla\left(\tau_{h, k} v\right)(z) \cdot\left(x_{0}^{z}-x_{0}\right) d z .
\end{aligned}
$$

Subtracting (19) from (18), we get

$$
\begin{aligned}
& v\left(x_{1}\right)+v\left(x_{3}\right)-2 v\left(x_{0}\right)= \\
& \quad-\frac{4}{h k} \int_{T_{1}^{\prime}} \frac{\left|x_{1}^{z}-x_{1}\right|}{\left|z-x_{1}\right|}\left[\nabla v(z)-\nabla\left(\tau_{h, k} v\right)(z)\right] \cdot\left(x_{1}^{z}-x_{1}\right) d z \\
& \quad+\frac{4}{h k} \int_{T_{1}} \frac{\left|x_{0}^{z}-x_{0}\right|}{\left|z-x_{0}\right|}\left[\nabla v(z)-\nabla\left(\tau_{h, k} v\right)(z)\right] \cdot\left(x_{0}^{z}-x_{0}\right) d z \\
& \quad \stackrel{\text { def }}{=} I_{1}+I_{2} .
\end{aligned}
$$


Consider $I_{1}$ the first integral in the right-hand side of (20). Thanks to (3), we have $\left|x_{1}^{z}-x_{1}\right| \leq C h$, for any $z \in T_{1}^{\prime}$. Therefore, we get

$$
\begin{aligned}
\left|I_{1}\right| & \leq C \int_{T_{1}^{\prime}} \frac{1}{\left|z-x_{1}\right|}\left|\nabla v(z)-\nabla\left(\tau_{h, k} v\right)(z)\right| d z \\
& \leq C h^{\alpha}\left(\int_{T_{1}^{\prime}} \frac{1}{\left|z-x_{1}\right|^{p^{\prime}}} d z\right)^{\frac{1}{p^{\prime}}}\left(\int_{T_{1}^{\prime}}\left|\frac{\nabla v(z)-\nabla\left(\tau_{h, k} v\right)(z)}{h^{\alpha}}\right|^{p} d z\right)^{\frac{1}{p}} \\
& \leq C h^{\alpha}\left(\int_{B_{h}} \frac{1}{\left.\left|z-x_{1}\right|\right|^{p^{\prime}}} d z\right)^{\frac{1}{p^{\prime}}}\left(\int_{T_{1}^{\prime}}\left|\frac{\nabla v(z)-\nabla\left(\tau_{h, k} v\right)(z)}{h^{\alpha}}\right|^{p} d z\right)^{\frac{1}{p}} \\
& \leq C h^{\frac{2}{p^{\prime}}-1+\alpha}\left(\int_{T_{1}^{\prime}}\left|\frac{\nabla v(z)-\nabla\left(\tau_{h, k} v\right)(z)}{h^{\alpha}}\right|^{p} d z\right)^{\frac{1}{p}},
\end{aligned}
$$

where $B_{h}$ is the ball of radius $\sqrt{h^{2}+k^{2}} \leq C h$ centered in $x_{1}$. The term $I_{2}$ in (20) is estimated in the same way, so that we finally have

$\left|v\left(x_{1}\right)+v\left(x_{3}\right)-2 v\left(x_{0}\right)\right| \leq C h^{\frac{2}{p^{\prime}}-1+\alpha}\left(\int_{\mathcal{K}^{*}}\left|\frac{\nabla v(z)-\nabla\left(\tau_{h, k} v\right)(z)}{h^{\alpha}}\right|^{p} d z\right)^{\frac{1}{p}}$.

Similarly, using Taylor expansions of $v$ between $x_{4}$ (respectively $x_{0}$ ) and the points of the segment $\left[d_{0} d_{0}^{\prime}\right]$ (see Figure 2), we get

$\left|v\left(x_{2}\right)+v\left(x_{4}\right)-2 v\left(x_{0}\right)\right| \leq C h^{\frac{2}{p^{\prime}}-1+\alpha}\left(\int_{\mathcal{K}^{*}}\left|\frac{\nabla v(z)-\nabla\left(\tau_{-h, k} v\right)(z)}{h^{\alpha}}\right|^{p} d z\right)^{\frac{1}{p}}$

where the translated function $\tau_{-h, k} v$ is defined by $\tau_{-h, k} v(z)=v(z+$ $\left.\left(x_{0}-x_{4}\right)\right)$. Using the two previous estimates, we get

$$
\begin{aligned}
& \frac{1}{h^{\alpha}}\left|\frac{v\left(x_{4}\right)-v\left(x_{3}\right)}{h}-\frac{v\left(x_{1}\right)-v\left(x_{2}\right)}{h}\right| \\
& \leq \frac{1}{h^{1+\alpha}}\left|\left[v\left(x_{4}\right)+v\left(x_{2}\right)-2 v\left(x_{0}\right)\right]-\left[v\left(x_{1}\right)+v\left(x_{3}\right)-2 v\left(x_{0}\right)\right]\right| \\
& \leq C h^{-\frac{2}{p}}\left(\int_{\mathcal{K}^{*}}\left|\frac{\nabla v(z)-\nabla\left(\tau_{h, k} v\right)(z)}{h^{\alpha}}\right|^{p} d z\right)^{\frac{1}{p}} \\
& \quad+C h^{-\frac{2}{p}}\left(\int_{\mathcal{K}^{*}}\left|\frac{\nabla v(z)-\nabla\left(\tau_{-h, k} v\right)(z)}{h^{\alpha}}\right|^{p} d z\right)^{\frac{1}{p}} .
\end{aligned}
$$


Hence, summing this inequality over $\mathcal{T}^{*}$, we deduce that

$$
\begin{aligned}
& \sum_{\mathcal{K}^{*} \in \mathcal{T}^{*}} m\left(\mathcal{K}^{*} \cap \Omega\right)\left|\frac{\delta_{3}^{\mathcal{K}^{*}}\left(v^{\mathcal{T}}\right)+\delta_{1}^{\mathcal{K}^{*}}\left(v^{\mathcal{T}}\right)}{h^{\alpha}}\right|^{p} \\
& \leq C \int_{\Omega}\left|\frac{\nabla v(z)-\nabla\left(\tau_{h, k} v\right)(z)}{h^{\alpha}}\right|^{p} d z+C \int_{\Omega}\left|\frac{\nabla v(z)-\nabla\left(\tau_{-h, k} v\right)(z)}{h^{\alpha}}\right|^{p} d z \\
& \leq C\|v\|_{B_{\infty}^{1+\alpha, p} .}^{p}
\end{aligned}
$$

The claim of the Lemma follows.

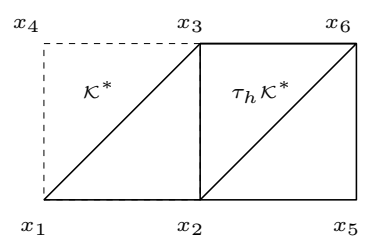

Fig. 3. Complete Besov norm estimate

Remark 6 Inequality (21) is in fact valid for any parallelogram $x_{1} x_{2} x_{3} x_{4}$, if we replace $\tau_{h, k} v$ and $\tau_{-h, k} v$ by suitable translations of $v$. Consider the situation described in Figure 3.

We can write

$$
\begin{aligned}
\frac{1}{h^{\alpha}}\left|\delta_{1}^{\tau_{h} \mathcal{K}^{*}}\left(v^{\mathcal{T}}\right)-\delta_{1}^{\mathcal{K}^{*}}\left(v^{\mathcal{T}}\right)\right|= & \frac{1}{h^{\alpha}}\left|\frac{v\left(x_{5}\right)-v\left(x_{2}\right)}{h}-\frac{v\left(x_{2}\right)-v\left(x_{1}\right)}{h}\right| \\
\leq & \frac{1}{h^{\alpha}}\left|\frac{v\left(x_{5}\right)-v\left(x_{2}\right)}{h}-\frac{v\left(x_{6}\right)-v\left(x_{3}\right)}{h}\right| \\
& +\frac{1}{h^{\alpha}}\left|\frac{v\left(x_{6}\right)-v\left(x_{3}\right)}{h}-\frac{v\left(x_{2}\right)-v\left(x_{1}\right)}{h}\right|,
\end{aligned}
$$

so that using (21) respectively in the square $x_{2} x_{5} x_{6} x_{3}$ and in the parallelogram $x_{1} x_{2} x_{6} x_{3}$, and summing over all the dual control volumes, we deduce an estimate for the complete Besov norm defined in (9):

$$
\llbracket v^{\mathcal{T}} \rrbracket_{1+\alpha, p, \mathcal{T}} \leq C\|v\|_{B_{\infty}^{1+\alpha, p}} .
$$

\subsection{Piecewise affine approximation of functions in $W_{0}^{1, p}$}

For any $u \in W_{0}^{1, p}(\Omega)$, let us denote by $\Pi_{\mathcal{T}} u$ the piecewise affine Lagrange interpolation of $u$ on the triangles constructed from the dual control volumes, as shown in Figure 4. 


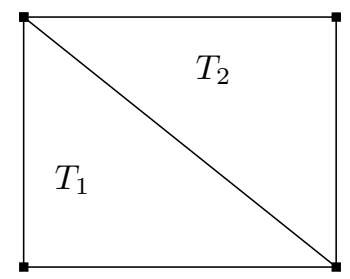

Interior dual control volume

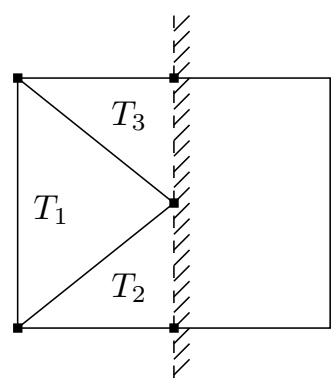

Boundary dual control volume

Fig. 4. Triangles in $\mathcal{K}^{*}$ for the Lagrange affine interpolation operator

The construction proposed on the boundary dual control volumes is chosen so that $\Pi_{\mathcal{T}} u$ lies in the space $W_{0}^{1, p}(\Omega)$. The corresponding interpolation error can be controlled as follows.

Proposition 1 For any $p>2$, there exists $C>0$ such that the following estimates hold:

$$
\begin{gathered}
\left\|u-\Pi_{\mathcal{T}} u\right\|_{W^{1, p}} \leq C\|u\|_{W^{1, p}}, \quad \forall u \in W_{0}^{1, p}(\Omega), \\
\left\|u-\Pi_{\mathcal{T}} u\right\|_{W^{1, p}} \leq C h\|u\|_{W^{2, p}}, \quad \forall u \in W_{0}^{1, p}(\Omega) \cap W^{2, p}(\Omega), \\
\left\|u-\Pi_{\mathcal{T}} u\right\|_{W^{1, p}} \leq C h^{\alpha}\|u\|_{\left.B_{\infty}^{1+\alpha, p}, \quad \forall \alpha \in\right] 0,1\left[, \forall u \in W_{0}^{1, p}(\Omega) \cap B_{\infty}^{1+\alpha, p}(\Omega) .\right.}
\end{gathered}
$$

Proof The first two estimates are classical results of the finite elements theory (see for example [7]). Note that the proof is straightforward in our case.

The third result follows directly from the first two estimates by applying the interpolation theorem associated to (5) for the operator Id $-\Pi_{\mathcal{T}}$.

\subsection{Proof of the error estimate}

We are now able to prove Theorem 2 .

We have $\nabla J_{\mathcal{T}}\left(u^{\mathcal{T}}\right)=0$, so that, thanks to Lemma 3 , we can write

$$
C\left\|u^{\mathcal{T}}-\bar{u}^{\mathcal{T}}\right\|_{1, p, \mathcal{T}}^{p} \leq J_{\mathcal{T}}\left(\bar{u}^{\mathcal{T}}\right)-J_{\mathcal{T}}\left(u^{\mathcal{T}}\right) .
$$

Let us rewrite this inequality under the form

$$
\begin{aligned}
C\left\|u^{\mathcal{T}}-\bar{u}^{\mathcal{T}}\right\|_{1, p, \mathcal{T}}^{p} \leq & {\left[J_{\mathcal{T}}\left(\bar{u}^{\mathcal{T}}\right)-J\left(\Pi_{\mathcal{T}} \bar{u}\right)\right]+\left[J\left(\Pi_{\mathcal{T}} \bar{u}\right)-J(\bar{u})\right] } \\
+ & {[J(\bar{u})-J(v)]+\left[J(v)-J_{\mathcal{T}}\left(u^{\mathcal{T}}\right)\right] }
\end{aligned}
$$


for any function $v \in W_{0}^{1, p}(\Omega)$. As $\bar{u}$ minimize $J$ over $W_{0}^{1, p}(\Omega)$, we see that the third term is negative. Now, we choose for $v$ the unique function which is affine on each triangles as shown in Figure 4 and such that $v\left(x_{\mathcal{K}}\right)=u_{\mathcal{K}}$. We denote this function by $v=\Pi_{\mathcal{T}} u^{\mathcal{T}}$. This notation is consistent with the one introduced in section 3.3, since we have for example $\Pi_{\mathcal{T}} \bar{u}=\Pi_{\mathcal{T}} \bar{u}^{\mathcal{T}}$. Therefore we can write

$$
\begin{aligned}
C\left\|u^{\mathcal{T}}-\bar{u}^{\mathcal{T}}\right\|_{1, p, \mathcal{T}}^{p} \leq & {\left[J_{\mathcal{T}}\left(\bar{u}^{\mathcal{T}}\right)-J\left(\Pi_{\mathcal{T}} \bar{u}^{\mathcal{T}}\right)\right] } \\
& +\left[J\left(\Pi_{\mathcal{T}} \bar{u}\right)-J(\bar{u})\right] \\
& +\left[J\left(\Pi_{\mathcal{T}} u^{\mathcal{T}}\right)-J_{\mathcal{T}}\left(u^{\mathcal{T}}\right)\right] .
\end{aligned}
$$

We recall the following classical result (see for example [6]):

Proposition 2 Suppose that $p>2$ and consider $\bar{u} \in W_{0}^{1, p}(\Omega)$ the solution to problem (1). There exists a constant $C>0$ such that for any $v \in W_{0}^{1, p}(\Omega)$,

$$
|J(v)-J(\bar{u})| \leq C\|v-\bar{u}\|_{W^{1, p}}^{2}\left(1+\|v\|_{W^{1, p}}+\|\bar{u}\|_{W^{1, p}}\right)^{p-2}
$$

Thanks to Proposition 2 and Proposition 1, the second term in (22) is majorated by

$$
\begin{aligned}
\left|J\left(\Pi_{\mathcal{T}} \bar{u}\right)-J(\bar{u})\right| & \leq C\left\|\Pi_{\mathcal{T}} \bar{u}-\bar{u}\right\|_{W^{1, p}}^{2}\left(\left\|\Pi_{\mathcal{T}} \bar{u}\right\|_{W^{1, p}}+\|\bar{u}\|_{W^{1, p}}\right)^{p-2} \\
& \leq C h^{\frac{2}{p-1}}\|\bar{u}\|_{B_{\infty}^{1+\frac{1}{p-1}}}^{2}\|\bar{u}\|_{W^{1, p}}^{p-2} .
\end{aligned}
$$

The first and the third terms in (22) are of the same kind. We have to estimate $\left|J\left(\Pi_{\mathcal{T}} v^{\mathcal{T}}\right)-J_{\mathcal{T}}\left(v^{\mathcal{T}}\right)\right|$ for $v^{\mathcal{T}} \in \mathbb{R}^{\mathcal{T}}$ satisfying a discrete Besov estimate.

The claim of Theorem 2 will follow, if we prove Lemma 6 below. Indeed, the desired estimate comes from (22), (23) and Lemmas 4, 5, 6.

Lemma 6 For any $\alpha \in] 0,1[$, there exists a constant $C>0$ such that for any $v^{\mathcal{T}} \in \mathbb{R}^{\mathcal{T}}$,

$$
\left|J\left(\Pi_{\mathcal{T}} v^{\mathcal{T}}\right)-J_{\mathcal{T}}\left(v^{\mathcal{T}}\right)\right| \leq C h^{2 \alpha}\left\|v^{\mathcal{T}}\right\|\left\|_{1+\alpha, p, \mathcal{T}}^{2}\right\| v^{\mathcal{T}}\left\|_{1, p, \mathcal{T}}^{p-2}+C h\right\| f\left\|_{L^{p^{\prime}}}\right\| v^{\mathcal{T}} \|_{1, p, \mathcal{T}}
$$


Proof By definition of $J$ and $J_{\mathcal{T}}$, we can write

$$
\begin{aligned}
& J\left(\Pi_{\mathcal{T}} v^{\mathcal{T}}\right)-J_{\mathcal{T}}\left(v^{\mathcal{T}}\right) \\
& =\frac{1}{p} \sum_{\mathcal{K}^{*} \in \mathcal{T}^{*}}\left(\int_{\mathcal{K}^{*} \cap \Omega}\left|\nabla \Pi_{\mathcal{T}} v^{\mathcal{T}}\right|^{p} d z-m\left(\mathcal{K}^{*} \cap \Omega\right)\left(B T_{\mathcal{K}^{*}}\left(v^{\mathcal{T}}\right), T_{\mathcal{K}^{*}}\left(v^{\mathcal{T}}\right)\right)^{\frac{p}{2}}\right) \\
& \quad-\sum_{\mathcal{K} \in \mathcal{T}}\left(\int_{\mathcal{K}} f(z) \Pi_{\mathcal{T}} v^{\mathcal{T}}(z) d z-m(\mathcal{K}) f_{\mathcal{K}} v_{\mathcal{K}}\right) \\
& \stackrel{\text { def }}{=} \frac{1}{p} \sum_{\mathcal{K}^{*} \in \mathcal{T}^{*}} I_{\mathcal{K}^{*}}+\sum_{\mathcal{K} \in \mathcal{T}} L_{\mathcal{K}} .
\end{aligned}
$$

Let us estimate each of the terms $I_{\mathcal{K}^{*}}$ and $L_{\mathcal{K}}$.

- Step 1: Take $\mathcal{K}^{*} \in \mathcal{T}^{*}$. We use here the notations of Figure 1. First assume that $\mathcal{K}^{*}$ is an interior dual control volume (see Figure 4). Dropping the superscripts $\mathcal{K}^{*}$, we have

$$
\nabla \Pi_{\mathcal{T}} v^{\mathcal{T}}(z)= \begin{cases}\left(\begin{array}{l}
\frac{v_{2}-v_{1}}{h} \\
\frac{v_{4}-v_{1}}{k}
\end{array}\right)=\left(\begin{array}{r}
\delta_{1} \\
-\delta_{4}
\end{array}\right), & \text { if } z \in T_{1}, \\
\left(\frac{v_{3}-v_{4}}{h}\right)=\left(\begin{array}{r}
-\delta_{3} \\
\frac{v_{3}-v_{2}}{k}
\end{array}\right), \quad \text { if } z \in T_{2} .\end{cases}
$$

Therefore

$$
\int_{\mathcal{K}^{*}}\left|\nabla \Pi_{\mathcal{T}} v^{\mathcal{T}}\right|^{p} d z=\frac{m\left(\mathcal{K}^{*}\right)}{2}\left(\left(\delta_{1}^{2}+\delta_{4}^{2}\right)^{\frac{p}{2}}+\left(\delta_{3}^{2}+\delta_{2}^{2}\right)^{\frac{p}{2}}\right) .
$$

It is an easy computation to see that if we define the following quadratic form in the variables $\left(\delta_{i}\right)_{i}$ :

$$
Q\left(\delta_{1}, \delta_{2}, \delta_{3}, \delta_{4}\right)=\frac{1}{2} \sum_{i=1}^{4} \delta_{i}^{2}+\xi\left(\frac{h}{k}\left(\delta_{1}+\delta_{3}\right)^{2}+\frac{k}{h}\left(\delta_{2}+\delta_{4}\right)^{2}\right),
$$

then we have

$$
\left|B^{\frac{1}{2}} T_{\mathcal{K}^{*}}\left(v^{\mathcal{T}}\right)\right|^{2}=Q\left(\delta_{1}, \delta_{2}, \delta_{3}, \delta_{4}\right), \quad \forall v^{\mathcal{T}} \in \mathbb{R}^{\mathcal{T}}
$$

By construction this quadratic form is consistent with $|\nabla \cdot|^{2}$ for affine functions on $\mathcal{K}^{*}$, that is to say that

$$
\forall a, b \in \mathbb{R}, \quad Q(a, b,-a,-b)=a^{2}+b^{2} .
$$

Consequently, (26) reads

$$
\int_{\mathcal{K}^{*}}\left|\nabla \Pi_{\mathcal{T}} v^{\mathcal{T}}\right|^{p} d z=\frac{m\left(\mathcal{K}^{*}\right)}{2}\left(Q\left(\delta_{1},-\delta_{4},-\delta_{1}, \delta_{4}\right)^{\frac{p}{2}}+Q\left(-\delta_{3}, \delta_{2}, \delta_{3},-\delta_{2}\right)^{\frac{p}{2}}\right) .
$$


Thus, we have to estimate

$$
\begin{aligned}
I_{\mathcal{K}^{*}}= & \int_{\mathcal{K}^{*}}\left|\nabla \Pi_{\mathcal{T}} v^{\mathcal{T}}\right|^{p} d z-m\left(\mathcal{K}^{*}\right)\left(B T_{\mathcal{K}^{*}}\left(v^{\mathcal{T}}\right), T_{\mathcal{K}^{*}}\left(v^{\mathcal{T}}\right)\right)^{\frac{p}{2}} \\
=m\left(\mathcal{K}^{*}\right)( & \frac{1}{2} Q\left(\delta_{1},-\delta_{4},-\delta_{1}, \delta_{4}\right)^{\frac{p}{2}}+\frac{1}{2} Q\left(-\delta_{3}, \delta_{2}, \delta_{3},-\delta_{2}\right)^{\frac{p}{2}} \\
& \left.\quad Q\left(\delta_{1}, \delta_{2}, \delta_{3}, \delta_{4}\right)^{\frac{p}{2}}\right)
\end{aligned}
$$

We denote by $\partial_{i} Q$ the derivative of $Q$ with respect to the $i$-th variable and for any multi-index $\alpha \in \mathbb{N}^{4}$, let $\partial^{\alpha} Q$ stand for the derivative $\partial_{1}^{\alpha_{1}} \partial_{2}^{\alpha_{2}} \partial_{3}^{\alpha_{3}} \partial_{4}^{\alpha_{4}} Q$

Set $\Delta_{1}=\left(\delta_{1},-\delta_{4},-\delta_{1}, \delta_{4}\right), \Delta_{2}=\left(-\delta_{3}, \delta_{2}, \delta_{3},-\delta_{2}\right)$ and $\Delta_{0}=$ $\left(\delta_{1}, \delta_{2}, \delta_{3}, \delta_{4}\right)$. Using Taylor expansions of $Q^{\frac{p}{2}}$ around the point $\Delta_{0}$, we get

$$
\begin{aligned}
& Q\left(\Delta_{1}\right)^{\frac{p}{2}}=Q\left(\Delta_{0}\right)^{\frac{p}{2}}-\frac{p}{2} Q^{\frac{p}{2}-1}\left(\Delta_{0}\right)\left(\partial_{2} Q\left(\Delta_{0}\right)\left(\delta_{2}+\delta_{4}\right)+\partial_{3} Q\left(\Delta_{0}\right)\left(\delta_{1}+\delta_{3}\right)\right) \\
& +\left(\int_{0}^{1} Q^{\frac{p}{2}-2}\left(\sum_{|\alpha|=1}\left|\partial^{\alpha} Q\right|^{2}+\sum_{|\beta|=2} Q\left|\partial^{\beta} Q\right|\right)\left(t \Delta_{1}+(1-t) \Delta_{0}\right) d t\right) \\
& \quad \times \mathcal{O}\left(\left(\delta_{1}+\delta_{3}\right)^{2}+\left(\delta_{2}+\delta_{4}\right)^{2}\right)
\end{aligned}
$$

and

$$
\begin{aligned}
& Q\left(\Delta_{2}\right)^{\frac{p}{2}}=Q\left(\Delta_{0}\right)^{\frac{p}{2}}-\frac{p}{2} Q^{\frac{p}{2}-1}\left(\Delta_{0}\right)\left(\partial_{1} Q\left(\Delta_{0}\right)\left(\delta_{1}+\delta_{3}\right)+\partial_{4} Q\left(\Delta_{0}\right)\left(\delta_{2}+\delta_{4}\right)\right) \\
& +\left(\int_{0}^{1} Q^{\frac{p}{2}-2}\left(\sum_{|\alpha|=1}\left|\partial^{\alpha} Q\right|^{2}+\sum_{|\beta|=2} Q\left|\partial^{\beta} Q\right|\right)\left(t \Delta_{2}+(1-t) \Delta_{0}\right) d t\right) \\
& \quad \times \mathcal{O}\left(\left(\delta_{1}+\delta_{3}\right)^{2}+\left(\delta_{2}+\delta_{4}\right)^{2}\right) .
\end{aligned}
$$

Consequently, we get

$$
\begin{aligned}
I_{\mathcal{K}^{*}}=- & m\left(\mathcal{K}^{*}\right)\left[\frac { p } { 4 } Q ^ { \frac { p } { 2 } - 1 } ( \Delta _ { 0 } ) \left(\left(\partial_{1} Q\left(\Delta_{0}\right)+\partial_{3} Q\left(\Delta_{0}\right)\right)\left(\delta_{1}+\delta_{3}\right)\right.\right. \\
& \left.+\left(\partial_{2} Q\left(\Delta_{0}\right)+\partial_{4} Q\left(\Delta_{0}\right)\right)\left(\delta_{2}+\delta_{4}\right)\right) \\
& \left.+\left(\left|\delta_{1}\right|+\left|\delta_{2}\right|+\left|\delta_{3}\right|+\left|\delta_{4}\right|\right)^{p-2} \mathcal{O}\left(\left(\delta_{1}+\delta_{3}\right)^{2}+\left(\delta_{2}+\delta_{4}\right)^{2}\right)\right] .
\end{aligned}
$$

But a straightforward computation using (27) shows that we have, under assumptions (3) and (13),

$$
\left|\partial_{1} Q\left(\Delta_{0}\right)+\partial_{3} Q\left(\Delta_{0}\right)\right|=\left|1+4 \xi \frac{h}{k}\right|\left|\delta_{1}+\delta_{3}\right| \leq C\left|\delta_{1}+\delta_{3}\right|,
$$


and

$$
\left|\partial_{2} Q\left(\Delta_{0}\right)+\partial_{4} Q\left(\Delta_{0}\right)\right|=\left|1+4 \xi \frac{k}{h}\right|\left|\delta_{2}+\delta_{4}\right| \leq C\left|\delta_{2}+\delta_{4}\right| .
$$

Hence, we deduce that

$$
\left|I_{\mathcal{K}^{*}}\right| \leq C m\left(\mathcal{K}^{*}\right)\left(\left|\delta_{1}\right|+\left|\delta_{2}\right|+\left|\delta_{3}\right|+\left|\delta_{4}\right|\right)^{p-2}\left(\left(\delta_{1}+\delta_{3}\right)^{2}+\left(\delta_{2}+\delta_{4}\right)^{2}\right) .
$$

In the case where $\mathcal{K}^{*}$ is a boundary control volume (for example near the right boundary of $\Omega$ - see the right part of Figure 4), we have

$$
\nabla \Pi_{\mathcal{T}} v^{\mathcal{T}}(z)=\left\{\begin{array}{l}
\left(\frac{0-\frac{v_{1}+v_{4}}{2}}{\frac{v_{4}-v_{1}}{k}}\right)=\left(\begin{array}{c}
\frac{\delta_{1}-\delta_{3}}{2} \\
-\delta_{4}
\end{array}\right), \quad \text { if } z \in T_{1}, \\
\left(\begin{array}{c}
\frac{0-v_{1}}{\frac{h}{2}} \\
0
\end{array}\right)=\left(\begin{array}{c}
-\delta_{1} \\
0
\end{array}\right), \quad \text { if } z \in T_{2} . \\
\left(\begin{array}{c}
\frac{0-v_{4}}{\frac{h}{2}} \\
0
\end{array}\right)=\left(\begin{array}{c}
\delta_{3} \\
0
\end{array}\right), \quad \text { if } z \in T_{3} .
\end{array}\right.
$$

Recall that in this boundary dual control volume $\mathcal{K}^{*}$ we have $\delta_{2}=\delta_{4}$. Using Taylor expansion of $Q^{\frac{p}{2}}$ around $\Delta_{0}$, we get

$$
\begin{aligned}
I_{\mathcal{K}^{*}}= & \frac{m\left(\mathcal{K}^{*} \cap \Omega\right)}{4} Q\left(\frac{\delta_{1}-\delta_{3}}{2}, \delta_{2}, \frac{\delta_{3}-\delta_{1}}{2},-\delta_{2}\right)^{\frac{p}{2}} \\
& +\frac{m\left(\mathcal{K}^{*} \cap \Omega\right)}{4} Q\left(\frac{\delta_{1}-\delta_{3}}{2},-\delta_{4}, \frac{\delta_{3}-\delta_{1}}{2}, \delta_{4}\right)^{\frac{p}{2}} \\
& +\frac{m\left(\mathcal{K}^{*} \cap \Omega\right)}{4} Q\left(\delta_{1}, 0,-\delta_{1}, 0\right)^{\frac{p}{2}}+\frac{m\left(\mathcal{K}^{*} \cap \Omega\right)}{4} Q\left(-\delta_{3}, 0, \delta_{3}, 0\right)^{\frac{p}{2}} \\
& -m\left(\mathcal{K}^{*} \cap \Omega\right) Q\left(\Delta_{0}\right)^{\frac{p}{2}} \\
= & -m\left(\mathcal{K}^{*} \cap \Omega\right)\left[\frac { p } { 4 } Q ^ { \frac { p } { 2 } - 1 } ( \Delta _ { 0 } ) \left(\left(\partial_{1} Q\left(\Delta_{0}\right)+\partial_{3} Q\left(\Delta_{0}\right)\right)\left(\delta_{1}+\delta_{3}\right)\right.\right. \\
& \left.+\left(\partial_{2} Q\left(\Delta_{0}\right)+\partial_{4} Q\left(\Delta_{0}\right)\right)\left(\delta_{2}+\delta_{4}\right)\right) \\
& \left.+\left(\left|\delta_{1}\right|+\left|\delta_{2}\right|+\left|\delta_{3}\right|+\left|\delta_{4}\right|\right)^{p-2} \mathcal{O}\left(\left(\delta_{1}+\delta_{3}\right)^{2}+\left(\delta_{2}+\delta_{4}\right)^{2}\right)\right] .
\end{aligned}
$$

We can conclude in the same way than in the case of an interior dual control volume. 
- Step 2: Take $\mathcal{K} \in \mathcal{T}$ and let us now deal with the term $L_{\mathcal{K}}=$ $\int_{\mathcal{K}} f(z)\left(\Pi_{\mathcal{T}} v^{\mathcal{T}}(z)-v_{\mathcal{K}}\right) d z$. Note that $v_{\mathcal{K}}=\Pi_{\mathcal{T}} v^{\mathcal{T}}\left(x_{\mathcal{K}}\right)$ so that we have,

$$
\begin{aligned}
\left|L_{\mathcal{K}}\right| & =\left|\sum_{\mathcal{K}^{*} \in V_{\mathcal{K}}} \int_{\mathcal{K}_{\mathcal{K}} *} f(z)\left(\Pi_{\mathcal{T}} v^{\mathcal{T}}(z)-\Pi_{\mathcal{T}} v^{\mathcal{T}}\left(x_{\mathcal{K}}\right)\right) d z\right| \\
& \leq C h \sum_{\mathcal{K}^{*} \in V_{\mathcal{K}}} \int_{\mathcal{K}_{\mathcal{K}^{*}}}|f(z)|\left|v^{\mathcal{T}}\right|_{1, \mathcal{K}^{*}} d z \\
& \leq C h \sum_{\mathcal{K}^{*} \in V_{\mathcal{K}}} m\left(\mathcal{K}^{*} \cap \Omega\right) g_{\mathcal{K}^{*}}\left|v^{\mathcal{T}}\right|_{1, \mathcal{K}^{*}}
\end{aligned}
$$

where for any $\mathcal{K}^{*} \in \mathcal{T}^{*}$, we define

$$
g_{\mathcal{K}^{*}}=\frac{1}{m\left(\mathcal{K}^{*} \cap \Omega\right)} \int_{\mathcal{K}^{*} \cap \Omega}|f(z)| d z .
$$

- Step 3: Note that we have

$$
\left(\sum_{\mathcal{K}^{*} \in \mathcal{T}^{*}} m\left(\mathcal{K}^{*} \cap \Omega\right)\left|g_{\mathcal{K}^{*}}\right|^{p^{\prime}}\right)^{\frac{1}{p^{\prime}}} \leq\left(\int_{\Omega}|f(z)|^{p^{\prime}} d z\right)^{\frac{1}{p^{\prime}}}=\|f\|_{L^{p^{\prime}}}
$$

by the Jensen inequality. Combining the estimates of the two previous steps and (24), by the Hölder inequality we get

$$
\begin{aligned}
& \left|J\left(\Pi_{\mathcal{T}} v^{\mathcal{T}}\right)-J_{\mathcal{T}}\left(v^{\mathcal{T}}\right)\right| \leq C \sum_{\mathcal{K}^{*} \in \mathcal{T}^{*}} I_{\mathcal{K}^{*}}+C \sum_{\mathcal{K} \in \mathcal{T}} L_{\mathcal{K}} \\
& \leq C h^{2 \alpha} \sum_{\mathcal{K}^{*} \in \mathcal{T}^{*}}\left|B^{\frac{1}{2}} T_{\mathcal{K}^{*}}\left(v^{\mathcal{T}}\right)\right|^{p-2}\left(\left|\frac{\delta_{1}+\delta_{3}}{h^{\alpha}}\right|^{2}+\left|\frac{\delta_{2}+\delta_{4}}{h^{\alpha}}\right|^{2}\right) \\
& \quad+C h \sum_{\mathcal{K}^{*} \in \mathcal{T}^{*}} m\left(\mathcal{K}^{*} \cap \Omega\right) g_{\mathcal{K}^{*}}\left|v^{\mathcal{T}}\right|_{1, \mathcal{K}^{*}} \\
& \leq C h^{2 \alpha}\left\|v^{\mathcal{T}}\right\|_{1, p, \mathcal{T}}^{p-2}\left\|v^{\mathcal{T}}\right\|_{1+\alpha, p, \mathcal{T}}^{2}+C h\|f\|_{L^{p^{\prime}}}\left\|v^{\mathcal{T}}\right\|_{1, p, \mathcal{T}} .
\end{aligned}
$$

\subsection{Improved estimates for Hölder data}

In the case where the data $f$ is supposed to lie in the Hölder space $\mathcal{C}^{0, \beta}(\bar{\Omega})$ with $\frac{1}{p-1}<\beta \leq 1$ we can improve our error estimate.

First of all, the control of the difference between $J$ and $J_{\mathcal{T}}$ (see Lemma 6) can be precised as follows: 
Lemma 7 Suppose that $f \in \mathcal{C}^{0, \beta}(\bar{\Omega})$, then for any $\left.\alpha \in\right] 0,1[$, there exists $C>0$ such that for any $v^{\mathcal{T}} \in \mathbb{R}^{\mathcal{T}}$, we have

$$
\begin{aligned}
& \left|J\left(\Pi_{\mathcal{T}} v^{\mathcal{T}}\right)-J_{\mathcal{T}}\left(v^{\mathcal{T}}\right)\right| \leq C h^{2 \alpha}\left\|v^{\mathcal{T}}\right\|_{1+\alpha, p, \mathcal{T}}^{2}\left\|v^{\mathcal{T}}\right\|_{1, p, \mathcal{T}}^{p-2} \\
& \quad+C h^{1+\alpha}\|f\|_{L^{p^{\prime}}} \llbracket v^{\mathcal{T}} \rrbracket_{1+\alpha, p, \mathcal{T}}+C h^{1+\beta}\|f\|_{\mathcal{C}^{0, \beta}}\left\|v^{\mathcal{T}}\right\|_{1, p, \mathcal{T}},
\end{aligned}
$$

where $\llbracket v^{\mathcal{T}} \rrbracket_{1+\alpha, p, \mathcal{T}}$ is the complete discrete Besov norm defined in (9).

Proof We follow the proof of Lemma 6 except that the Hölder regularity of $f$ give us a more precise estimate of the term $L_{\mathcal{K}}$ in $(24)$.

Indeed, let $\mathcal{K} \in \mathcal{T}$ be any control volume and let us choose $\mathcal{L}^{*} \in V_{\mathcal{K}}$. Let $\phi$ be the unique affine function on the whole control volume $\mathcal{K}$ such that $\phi\left(x_{\mathcal{K}}\right)=v_{\mathcal{K}}$ and $\nabla \phi=\left(\delta_{1}^{\mathcal{L}^{*}}\left(v^{\mathcal{T}}\right), \delta_{2}^{\mathcal{L}^{*}}\left(v^{\mathcal{T}}\right)\right)^{t}$. We can control the term $L_{\mathcal{K}}$ as follows:

$$
\begin{aligned}
\left|L_{\mathcal{K}}\right| \leq & \left|\int_{\mathcal{K}} f(z)\left(\Pi_{\mathcal{T}} v^{\mathcal{T}}(z)-\phi(z)\right) d z\right|+\left|\int_{\mathcal{K}} f(z)\left(\phi(z)-\phi\left(x_{\mathcal{K}}\right)\right) d z\right| \\
= & \left|\int_{\mathcal{K}} f(z)\left(\Pi_{\mathcal{T}} v^{\mathcal{T}}(z)-\phi(z)\right) d z\right| \\
& \quad+\left|\int_{\mathcal{K}}\left(f(z)-f\left(x_{\mathcal{K}}\right)\right)\left(\phi(z)-\phi\left(x_{\mathcal{K}}\right)\right) d z\right|
\end{aligned}
$$

since $\int_{\mathcal{K}}\left(\phi(z)-\phi\left(x_{\mathcal{K}}\right)\right) d z=0$, the point $x_{\mathcal{K}}$ being the center of $\mathcal{K}$. Since $\Pi_{\mathcal{T}} v^{\mathcal{T}}-\phi$ is affine by parts and vanishes in $x_{\mathcal{K}}$ we have for any $z \in \mathcal{K}$

$\left|\Pi_{\mathcal{T}} v^{\mathcal{T}}(z)-\phi(z)\right|=\left|z-x_{\mathcal{K}}\right|\left|\nabla \Pi_{\mathcal{T}} v^{\mathcal{T}}(z)-\nabla \phi(z)\right| \leq C h\left|\nabla \Pi_{\mathcal{T}} v^{\mathcal{T}}(z)-\nabla \phi\right|$.

Hence, the first term in (29) is controlled by

$$
\begin{aligned}
& C h \int_{\mathcal{K}}|f(z)|\left|\nabla \Pi_{\mathcal{T}} v^{\mathcal{T}}(z)-\nabla \phi\right| d z \\
& \quad \leq C m(\mathcal{K}) h\left|f_{\mathcal{K}}\right| \sum_{\mathcal{K}^{*} \in V_{\mathcal{K}}} \sum_{i=1}^{4}\left|\delta_{i}^{\mathcal{K}^{*}}\left(v^{\mathcal{T}}\right)-\delta_{i}^{\mathcal{L}^{*}}\left(v^{\mathcal{T}}\right)\right| .
\end{aligned}
$$

Thanks to the Hölder regularity of $f$, the second term in (29) is estimated by

$$
h^{3+\beta}\|f\|_{\mathcal{C}^{0, \beta}}\left|v^{\mathcal{T}}\right|_{1, \mathcal{L}^{*}}
$$

Summing these estimates over all the control volumes $\mathcal{K}$ and using the Hölder inequality gives exactly the claim. 
Notice that the complete discrete Besov norm can be controlled in the same way than $\left\|v^{\mathcal{T}}\right\|_{1+\alpha, p, \mathcal{T}}$ when $v^{\mathcal{T}}=u^{\mathcal{T}}$ or $v^{\mathcal{T}}=\bar{u}^{\mathcal{T}}$ as shown in the Remarks 5 and 6 . Hence, the Lemma above implies the improved estimate stated in Remark 3.

Let us now prove a new regularity result for the solution to (1) when the data enjoys Hölder regularity.

Lemma 8 If $\frac{1}{p-1}<\beta \leq 1$ and $f \in \mathcal{C}^{0, \beta}(\bar{\Omega})$, then the solution $\bar{u}$ to (1) satisfies

$$
\bar{u} \in B_{\infty}^{1+\frac{1+\beta}{p}, p}(\Omega)
$$

and

$$
\|\bar{u}\|_{B_{\infty}^{1+\frac{1+\beta}{p}, p}} \leq C\|f\|_{\mathcal{C}^{0, \beta}}^{\frac{1}{p}}\|f\|_{L^{p^{\prime}}}^{\frac{1}{p(p-1)}} .
$$

Proof As we have seen before, by extending $\bar{u}$ and $f$ to a larger domain, it is enough to consider the case of periodic boundary conditions. Let $r \in \mathbb{R}^{2}$ given and $\tau_{r}$ be the translation operator associated to $r$. We have

$$
-\operatorname{div}\left(\left|\nabla \tau_{r} \bar{u}\right|^{p-2} \nabla \tau_{r} \bar{u}\right)+\operatorname{div}\left(|\nabla \bar{u}|^{p-2} \nabla \bar{u}\right)=\tau_{r} f-f,
$$

so that multiplying by $\tau_{r} \bar{u}-\bar{u}$ and integrating over a periodicity cell $\Omega$ we get

$$
\begin{aligned}
\int_{\Omega}\left|\nabla \tau_{r} \bar{u}-\nabla \bar{u}\right|^{p} d z & \leq|r|^{\beta}\|f\|_{\mathcal{C}^{0, \beta}} \int_{\Omega}\left|\tau_{r} \bar{u}-\bar{u}\right| d z \\
& \leq|r|^{\beta}|r|\|f\|_{\mathcal{C}^{0, \beta}}\|\nabla \bar{u}\|_{L^{1}} \leq C|r|^{1+\beta}\|f\|_{\mathcal{C}^{0, \beta}}\|\bar{u}\|_{W^{1, p}} \\
& \leq C|r|^{1+\beta}\|f\|_{\mathcal{C}^{0, \beta}}\|f\|_{L^{p^{\prime}}}^{\frac{1}{p-1}} .
\end{aligned}
$$

Hence, the result follow by the characterization (6) of the Besov space $B_{\infty}^{1+\frac{1+\beta}{p}, p}$.

As usual, we have a discrete adaptation of this result which reads:

Lemma 9 If $\frac{1}{p-1}<\beta \leq 1$ and $f \in \mathcal{C}^{0, \beta}(\bar{\Omega})$, then the approximate solution $u^{\mathcal{T}}$ satisfies

$$
\|\| u^{\mathcal{T}}\left\|_{1+\frac{1+\beta}{p}, p, \mathcal{T}} \leq C\right\| f\left\|_{\mathcal{C}^{0, \beta}}^{\frac{1}{p}}\right\| f \|_{L^{p^{\prime}}}^{\frac{1}{p(p-1)}} .
$$

The proof is adapted from the one of Lemma 8 as we did in the proof of Lemma 4.

We can now state the following improved error estimate result. 
Theorem 3 Let $\left.\beta \in] \frac{1}{p-1}, 1\right]$. There exists a constant $C>0$ depending only on $c_{1}$ in (3) and $\gamma$ in (13), such that for any $f \in \mathcal{C}^{0, \beta}(\bar{\Omega})$, the solution $\bar{u}$ of (1) and $u^{\mathcal{T}}$ solution of the scheme (10) satisfy

$$
\left\|u^{\mathcal{T}}-\bar{u}^{\mathcal{T}}\right\|_{1, p, \mathcal{T}} \leq C h^{\frac{2(1+\beta)}{p^{2}}}\|f\|_{\mathcal{C}^{0, \beta}}^{\frac{1}{p-1}}
$$

Since $\beta>\frac{1}{p-1}$ in this last result, the convergence rate obtained here is obviously better than the one given by Theorem 2 .

Proof Using the new estimates above, the proof is very similar to the one of Theorem 2.

We consider the main inequality (22). Under the assumptions of the theorem, the second term in this inequality is estimated by using Propositions 1 and 2 as well as Lemma 8. The first and third terms in (22) are controled by using Lemmas 7, 8, 4 and 9.

\section{Numerical results}

We claimed in the introduction that Theorem 1 is optimal. Indeed, for $p>2$ consider a regular function $\rho$ with compact support in $\Omega=]-1,1[\times]-1,1[$ such that $\rho=1$ in a neighborhood of the point $(0,0)$. For $\alpha, \beta \in \mathbb{R}$, set

$$
u_{\alpha, \beta}(z)=\rho(z)|z|^{\alpha}(-\log |z|)^{\beta}, \quad \forall z \in \Omega .
$$

Evaluating the p-laplacian of $u_{\alpha, \beta}$, we have

$$
f_{\alpha, \beta} \stackrel{\text { def }}{=}-\operatorname{div}\left(\left|\nabla u_{\alpha, \beta}\right|^{p-2} \nabla u_{\alpha, \beta}\right) \underset{|z| \rightarrow 0}{\sim} C|z|^{(\alpha-1)(p-1)-1}(-\log |z|)^{\beta(p-1)} .
$$

Consequently, if we choose

$$
\alpha=\frac{p-1}{p}+\frac{1}{p(p-1)} \text { and } \beta=-\frac{2}{p},
$$

then $f_{\alpha, \beta}$ lies in $L^{p^{\prime}}(\Omega)$ (but not in $L^{q}(\Omega)$ for any $q>p^{\prime}$ ). Furthermore, following [9, p 43], for such $(\alpha, \beta)$ the function $u_{\alpha, \beta}$ is not in $B_{r}^{s, p}(\Omega)$ for any $r \in[1,+\infty]$ and any $s>1+\frac{1}{p-1}$.

Using this example of a solution with critical regularity for the problem, we can perform numerical tests in order to show that our theoretical results provide sharp convergence order. For each $p \in$ $\{3.0,3.5,4.0\}$, we define $\alpha$ and $\beta$ by (30) and we compare (for various meshes) the exact solution $u_{\alpha, \beta}$ with the approximate solution obtained with data $f_{\alpha, \beta}$. 


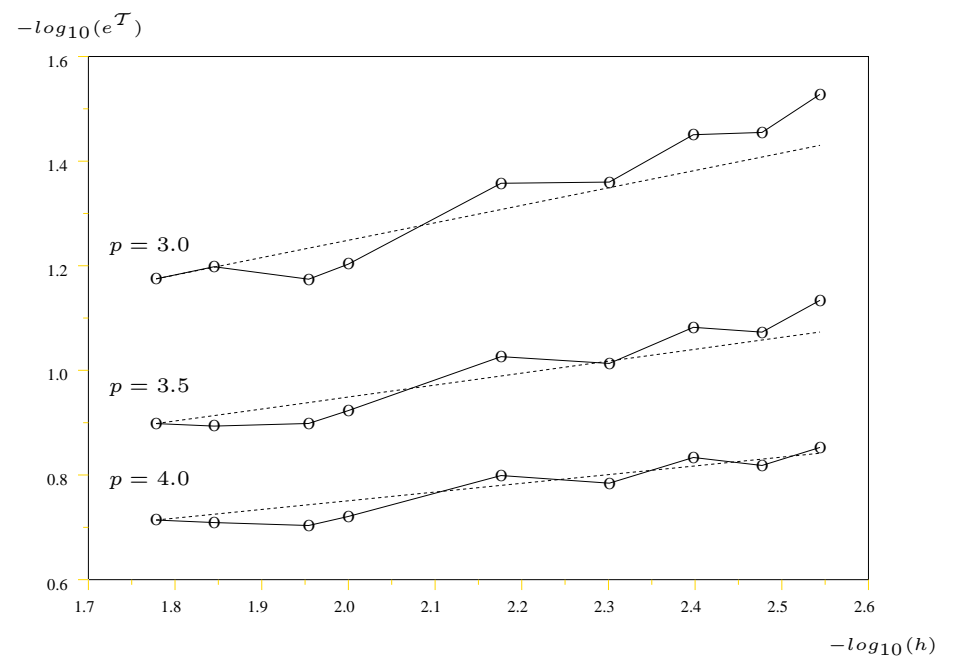

Fig. 5. Numerical results for $p \in\{3.0,3.5,4.0\}$

The results are summed up in Figure 5 where we plot in logarithmic scale the relative error $e^{\mathcal{T}}=\frac{\left\|u^{\mathcal{T}}-\bar{u}^{\mathcal{T}}\right\|_{1, p, \mathcal{T}}}{\left\|\bar{u}^{\mathcal{T}}\right\|_{1, p, \mathcal{T}}}$ in front of the size of the mesh $h$. We have also plotted for each value of $p$, a dotted straight line with slope $\frac{2}{p(p-1)}$ which is exactly the convergence order that we obtained in Theorem 2.

We can see that the numerical results are in good accordance with the predicted convergence rate. Hence, our theoretical results seems to be optimal within the class of $L^{p^{\prime}}$ data.

\section{Conclusion}

In this paper we proved an optimal error bound in the discrete $W^{1, p}$ norm for the finite volume approximation of the $p$-laplacian $(p>2)$ with general $L^{p^{\prime}}(\Omega)$ data on uniform cartesian grids. In this case where the exact solution does not belong to $W^{2, p}(\Omega)$ but only to the Besov space $B_{\infty}^{1+\frac{1}{p-1}, p}(\Omega)$, classical finite volume techniques can not be used to estimate the consistency error of numerical fluxes. Hence, our proof consists in taking advantage of the variationnal structure of the continuous problem and of its discrete approximation. The error estimate follows if one shows a discrete Besov estimate independent of the mesh size for the approximate solution but also for the projection of the exact solution on the space of piecewise constant functions. 
We provide in this paper a proof of these two bounds in the case of uniform cartesian grids which implies the error estimates. Numerical results for some analytical solutions with critical regularity give some evidences that the convergence rate obtained is optimal.

In a forthcoming work, we address the problem of proving these discrete Besov estimates for more general cartesian grids, in particular for meshes obtained by taking the image of uniform grids through smooth enough maps. In this framework, the control volumes can be chosen finer near the singularities of the data. To our knowledge, the adaptation of our approach to general non-cartesian grids (see for instance [2]) is an open question at the present time.

\section{References}

1. B. Andreinnov, F. Boyer And F. Hubert, Finite volume schemes for the p-laplacian on cartesian meshes, M2AN Math. Model. Numer. Anal., 38, $\mathrm{n}^{\circ}$ 6, pp. 931-960, 2004.

2. B. Andreianov, F. Boyer And F. Hubert, "Duplex" finite volume schemes for nonlinear elliptic problems on general 2D meshes, to appear in Finite Volumes for Complex Applications, IV (Marrakech), 2005.

3. B. Andreinanov, M. Gutnic and P. Wittbold, Convergence of finite volume approximations for a nonlinear elliptic-parabolic problem : a "continuous" approach, SIAM J. on Numer. Anal., 42 no 1, pp. 228-251, 2004.

4. J.W. BARRETT AND W.B. LiU, A remark on the regularity of the solutions of the $p$-laplacian and its application to the finite element approximation, Journal of Math. anal. Appl. 178, pp. 470-487, 1993.

5. J.W. BARRETT AND W.B. LiU, Finite element approximation of the $p$ laplacian, Math. Comp. 61, pp. 523-537, 1993.

6. S. CHOw, Finite element error estimates for non-linear elliptic equations of monotone type, Numer. Math. 54 , pp. 373-393, 1989.

7. P.G. Ciarlet, The finite element method for elliptic problems, Studies in Mathematics and its Applications, Vol. 4, North-Holland publishing company, 1978.

8. R. Glowinski And A. Marrocco, Sur l'approximation par éléments finis d'ordre un, et la résolution, par pénalisation-dualité, d'une classe de problèmes de Dirichlet non linéaires, Journal of Math. anal. Appl. RAIRO Anal. Num. 2, pp. 41-64, 1975.

9. T. Runst And W. Sickel, Sobolev spaces of fractional order, Nemytskij operators and nonlinear partial differential equations, De Gruyter series in nonlinear analysis and applications, De Gruyter, 1996.

10. J. Simon, Caractérisation d'espaces fonctionnels, Bollettino U. M.I. 15-B , pp. 687-714, 1978.

11. J. Simon, Régularité de la solution d'un problème aux limites non linéaires, Ann. Fac. Sciences Toulouse 3, pp. 247-274, 1981.

12. H. TRIEBEL, Interpolation theory, function spaces, differential operators, North-Holland, Amsterdam, 1978. 
13. V.B. Tyukthin, The rate of convergence of approximation methods for solving one-sided variational problems. I, Teoret. Mat. Fiz. $51 \mathrm{n}^{\circ}$ 2, pp 111-113, 121,1982 . 